\title{
A revised radiometric calibration for the Hinode/EIS instrument ${ }^{\star}$
}

\author{
G. Del Zanna
}

\author{
DAMTP, Centre for Mathematical Sciences, Wilberforce road Cambridge, CB3 OWA, UK \\ e-mail: g.del.zanna@damtp.cam.ac.uk
}

Received 28 November 2012 / Accepted 3 May 2013

\begin{abstract}
An assessment of the in-flight radiometric calibration of the Hinode EUV Imaging Spectrometer (EIS) is presented. This is done with the line ratio technique applied to a wide range of observations of the quiet Sun, active regions, and flares from 2006 until 2012. The best diagnostic lines and the relevant atomic data are discussed in detail. Radiances over the quiet Sun are also considered, with comparisons with previous measurements. Some departures in the shapes of the ground calibration responsivities are found at the start of the mission. These shapes do not change significantly over time, with the exception of the shorter wavelengths of the EIS short-wavelength (SW) channel, which shows some degradation. The sensitivity of the SW channel at longer wavelengths does not show significant degradation, while that of the long-wavelength (LW) channel shows a significant degradation with time. By the beginning of 2010 the responsivity of the LW channel was already lower than the values measured on the ground by a factor of two or more. A first-order correction is proposed. With this correction, the main ratios of lines in the two channels become constant to within a relative $20 \%$, and the He II $256 \AA$ radiances over the quiet Sun also become constant over time. This correction removes long-standing discrepancies for a number of lines and ions, in particular those involving the strongest Fe X, Fe XIII, Fe XIV, Fe XVII, and Fe XXIV lines, where discrepancies of factors of more than two were found. These results have important implications for various EIS science analyses, in particular for measurements of temperatures, emission measures and elemental abundances.
\end{abstract}

Key words. atomic data - techniques: spectroscopic - Sun: abundances - Sun: corona - line: identification

\section{Introduction}

The Hinode EUV Imaging Spectrometer (EIS, see Culhane et al. 2007) has been observing emission lines from a wide range of ions since December 2006; its two wavelength bands ( $S W$ : 166-212 ̊; $L W: 245-291 \AA$ ) provide detailed measurements of electron densities and temperatures, as well as emission measures and elemental abundances. For these measurements an accurate radiometric calibration is of paramount importance. Hinode EIS was radiometrically calibrated on the ground at the Rutherford Appleton Laboratory (RAL, UK) (Lang et al. 2006), providing an overall uncertainty of about $20 \%$. It has been generally assumed that relative line intensities are measured by EIS with greater accuracy.

A number of problems in the EIS intensities have been highlighted over the years, however, with discrepancies, typically of a factor of two, that could only be ascribed to calibration problems. Significant discrepancies $(50 \%)$ were already found in the 2007 August observations of the strong Fe XIII lines in the LW channel around $250 \AA$ (Del Zanna 2011, 2012). They could not be ascribed to problems such as blending or the atomic data, given that excellent agreement with the Malinovsky \& Heroux (1973; hereafter MH73) spectrum was found, for example. Significant discrepancies were also found in a few of the strong Fe XIV lines (Del Zanna 2012). Again, they could only be ascribed to calibration problems.

Significant discrepancies in the Fe XVII 204.6 and $254.9 \AA$ ratio were reported by Del Zanna (2008) and Del Zanna \& Ishikawa (2009). These problems are not present in previous (Skylab) observations nor in laboratory measurements, where

* Appendices are available in electronic form at http: //www . aanda.org good agreement with theory is found (Watanabe, priv. comm.). The Fe XVII lines form a branching ratio, for which atomic data are very accurate.

Significant discrepancies in the two strongest flare diagnostic lines for EIS, the Fe XXIV 192.0 and $255.1 \AA$ lines, were also reported by Del Zanna (2008). These discrepancies are present in the literature, although often not specifically mentioned. For example, Hara et al. (2011) presents a 2007 May 19 observation where the doublet ratio is approximately 2.9 photons, instead of the expected (and well-known) value of 1.85. Recent flare observations in 2012 (discussed below) have presented discrepancies of more than a factor of two for both Fe XVII and Fe XXIV ratios, suggesting that the discrepancy in the relative calibration between the SW and LW channels has increased over time. Problems in this important ratio have recently been confirmed by other authors (Young et al. 2013; Hara, priv. comm.).

Wang et al. (2011) performed a direct comparison between EIS SW and EUNIS quiet Sun (QS) observations in 2007, finding a small (20\%) decrease compared to the ground calibration, a variation within the combined EUNIS and EIS uncertainties. An estimate of the relative responsivity at a few wavelengths in the LW channel was also performed, finding again overall consistency, but with larger uncertainties.

Starting in December 2006, regular observations of quiet Sun regions near Sun center have been taken to monitor EIS sensitivity changes. The initial studies (SYNOP001 and SYNOP002) downloaded the full EIS spectral range. However, after the $X$-band transmitter failure early in 2008 , these were replaced with studies that only telemetred a limited number of emission lines. The radiances of the He II $256 \AA$ line show a clear decrease over time, and a preliminary long-term correction for all the EIS wavelengths was proposed, assuming that 
the He II radiances should stay constant over time (Mariska, priv. comm.). This was implemented in the EIS software. The sensitivity decay was modelled as an exponential decay of the form $\mathrm{e}^{-\mathrm{t} / \tau}$, where $\mathrm{t}$ is the time of the observation in days since the Hinode launch (2006 September 22 21:36 UT), and $\tau$ is the decay time, 1894 days (see EIS Software Notes \#1,2).

Kamio \& Mariska (2012) have recently presented QS radiances in EIS lines obtained from these synoptic studies. The EIS radiances were corrected for the sensitivity decay using an improved curve based on the He II radiances: $\left(\mathrm{e}^{-\mathrm{t} / \tau_{1}}+\right.$ $\left.\mathrm{e}^{-\mathrm{t} / \tau_{2}}\right) / 2$, where the e-folding times $\tau_{1}$ and $\tau_{2}$ are 467 and 11311 days, respectively. This correction was applied to both SW and LW channels, since no evidence for a variation between the SW and LW channels was found. The EIS radiances in lines formed above $1 \mathrm{MK}$ show clear solar cycle trends, and the He II radiances become constant after the correction. However, low-temperature lines such as Fe VIII $185.2 \AA$ and Si VII $275.3 \AA$ show unrealistic increases (see Del Zanna et al. 2010a; Del Zanna \& Andretta 2011 for a discussion on solar cycle effects on spectral lines), a clear indication of a problem in the correction.

The He II $256 \AA$ is the strongest LW line in QS observations, and should stay relatively constant, although as shown in Del Zanna et al. (2010a) and Del Zanna \& Andretta (2011), the radiances of the helium lines are affected along the solar cycle by the coronal radiation, possibly because of photoionisationrecombination effects (see references in Andretta et al. 2003). Large variations between quiet Sun and coronal hole areas are also present in helium lines. So, the use of the He II $256 \AA$ for calibration is not ideal (see below for further complications because of blending). It should be accurate, however, if proper QS observations are selected and the line carefully deblended.

Mariska (2013) re-analysed the set of synoptic observations, noting that the radiances in the Fe VIII 185.2 $\AA$, Si VII $275.3 \AA$, and Fe X $184.5 \AA$ lines decreased in a similar way. The average decrease is small, of the order of $25 \%$, corresponding to an e-folding time of $7358 \pm 1030$ days. The He II $256 \AA$ has a completely different behaviour, a nearly linear drop of a factor of two in the first two years of the mission, followed by a slower decay. The He II is severely blended, but these blends cannot explain the difference. Mariska (2013) suggested that the He II should be discarded, and that the results of the three above-mentioned lines indicate a slow decrease of both SW and LW channels over time.

A more complete analysis of the in-flight changes of the EIS responsivities is, in principle, possible using line ratios and the full EIS spectral range, and not just line radiances. The aim of this paper is to present preliminary results from such an analysis. Similar procedures adopted for the in-flight calibration of the Solar and Heliospheric Observatory (SOHO) Coronal Diagnostic Spectrometer (CDS) normal-incidence (NIS) and grazing-incidence (GIS) channels (Del Zanna et al. 2001, 2010a) are followed here. Del Zanna et al. (2001) used the line ratio technique, described in more detail below, while Del Zanna et al. (2010a) showed that the radiances of low transition-region lines are relatively unchanged along the solar cycle and can be used to correct for the long-term degradation. These corrections have been adopted by the CDS team to produce the final calibrated NIS data for the whole SOHO mission.

The present analysis has only been made possible now that we have a more complete understanding of the line identifications and the atomic data. Over the past ten years $w^{1}$ have

\footnotetext{
UK APAP network http://www . apap-network .org
}

calculated the atomic data for the main EIS lines and have provided them to the community via the CHIANTI database ${ }^{2}$ (Dere et al. 1997; Landi et al. 2012). Recently, we have performed large-scale R-matrix calculations for several ions to address the missing data problem of the soft X-ray lines. The calculations for Fe X (Del Zanna et al. 2012b), Fe XI (Del Zanna \& Storey 2013), and Fe XII (Del Zanna et al. 2012a) are particularly relevant here because they resolved long-standing discrepancies with observations for a few among the strongest EUV lines. These data have been used in the present analysis and will be made available in a future CHIANTI release.

A review of line identifications and atomic data for all the EIS low-temperature lines was presented in Del Zanna (2009a). A review of line identifications and atomic data for all the EIS coronal lines was presented in Del Zanna (2012). The main flare lines were discussed in Del Zanna (2008), Del Zanna et al. (2011), and Del Zanna \& Ishikawa (2009). Several dozen new lines have been identified, while similar numbers still await identification. Most of the EIS spectral lines, despite the high spectral resolution, turned out to be blended, and for this calibration work a careful selection of the lines and observations needed to be done.

\section{The method and the data}

The method consists of choosing the appropriate observations and line ratios to constrain the relative responsivities of the instrument (Del Zanna et al. 2001). The method has been applied in many instances, not only for the SOHO CDS. For example, Neupert \& Kastner (1983) used this method for an in-flight calibration of the OSO V and OSO VII EUV spectrometers. Brosius et al. (1998) also used the same method to calibrate observations from the Solar EUV Rocket Telescope and Spectrometer in 1995 (SERTS-95). Line ratios were also used by Young et al. (1998) to indicate problems in the calibration of the SERTS-89 active region spectrum (Thomas \& Neupert 1994).

There are several good line ratios that can be used to check the in-flight relative calibration, as detailed below. The best are branching ratios, with typical uncertainties of $10 \%$ or less. But there are also a number of ratios that are useful for the calibration, i.e. those that are largely independent of density and temperature. Each ratio was assessed against various atomic calculations and observations. The best calibrated EUV spectrum is that of MH73, which shows typical agreement with theory with remarkably few percentage points.

The SW and LW channels have a sufficient number of line ratios to check the shape of their effective areas. The major difficulty has been assessing the relative calibration between the two channels because very few line ratios are available. Another significant problem affects the use of data prior to 2008 Aug. 24 (when the grating focus was adjusted). Until this date, there was an offset of about $2^{\prime \prime}$ in the pointing of the SW and LW channels, meaning that the observations in the two channels were not simultaneous. Since most observations were made with the $1^{\prime \prime}$ slit, it took more than twice the exposure time to observe the same region. Most synoptic observations are single-slit observations, and there is no way to correct for this. Whenever raster observations were available, co-spatial regions have been selected for the present work. The lack of co-spatiality limits the use of data prior to 2008 August, in particular for the Fe XVII and Fe XXIV flare lines, since temporal variability is often significant on the exposure time scales.

\footnotetext{
2 http://www.chiantidatabase.org
} 
Table 1. Line ratios used for the EIS calibration.

\begin{tabular}{|c|c|c|c|c|c|c|}
\hline Line ratio $(\AA)$ & $\begin{array}{c}\text { Predicted } \\
(\log \mathrm{Ne}=8-9)\end{array}$ & MH73 & $\begin{array}{c}\text { Observed } \\
\mathrm{DN} / \mathrm{s}\end{array}$ & $\begin{array}{l}R_{\text {eff }} \\
\text { Pres. }\end{array}$ & $\begin{array}{c}R_{\mathrm{eff}} \\
\text { Ground } \\
\end{array}$ & Det. \\
\hline Fe VIII 185.2/196.0 & $4.5-5.3(5.3,20 \%)$ & & $1.23(0.10,1.3 / 1.1)$ & 0.206 & 0.256 & SW \\
\hline Fe IX $189.94 / 197.85$ & $0.78-1.0(1.0,30 \%)$ & & $0.68(0.04,1.6 / 2.4)$ & 0.653 & 0.704 & SW \\
\hline Fe X 174.5/184.5 & $4.52-4.32(4.4,10 \%)$ & $4.53(2 \%)$ & $0.10(0.01,0.3 / 3.4)$ & 0.021 & 0.024 & SW \\
\hline Fe X 177.2/184.5 & $2.6-2.49(2.55,10 \%)$ & $2.53(0 \%)$ & $0.16(0.01,0.5 / 3.4)$ & 0.060 & 0.074 & SW \\
\hline Fe X 184.5/190.0 (bl) & $2.94(30 \%)$ & (bl) & $0.92(0.05,3.4 / 3.7)$ & 0.304 & 0.332 & BR SW \\
\hline Fe X 207.4/184.5 & $0.14-0.18(0.15,30 \%)$ & & $0.05(0.01,0.2 / 3.4)$ & 0.375 & 0.321 & SW \\
\hline Fe X $257.3(\mathrm{sbl}) / 184.5$ & $1.52-1.12$ & & $0.77(0.08,7.5 / 9.8)$ & 0.706 & 0.928 & LW/SW \\
\hline Fe XI 188.2/192.8 (bl) & 4.8 & & $2.1(0.1,42.5 / 20)$ & 0.427 & 0.559 & BR SW \\
\hline Fe XI 202.7/188.3 & $0.1(30 \%)$ & & $0.06(0.01,0.4 / 7.0)$ & 0.648 & 0.422 & BR SW \\
\hline Fe XI 178.1/182.2 & 0.27 & & $0.07(0.01,0.4 / 5.5)$ & 0.254 & 0.231 & BR SW \\
\hline Fe XI 180.4/188.2 & $2.0(20 \%)$ & & $0.34(0.01,2.4 / 7.0)$ & 0.105 & 0.114 & SW \\
\hline Fe XI $257.5(\mathrm{sbl}) / 188.2$ & 0.165 & & $0.05(0.006,2.1 / 42.5)$ & 0.411 & 0.413 & LW/SW \\
\hline Fe XII 192.4/195.1 (sbl) & 0.315 & $0.315(0 \%)$ & $0.24(0.01,5.7 / 23.4)$ & 0.751 & 0.844 & SW \\
\hline Fe XII 193.5/195.1 (sbl) & 0.67 & $0.67(0 \%)$ & $0.59(0.01,13.9 / 23.4)$ & 0.873 & 0.924 & SW \\
\hline Fe XII 186.9 (sbl)/196.6 (bl) & $3.45-3.7(3.5,20 \%)$ & & $1.04(0.11,1.5 / 1.5)$ & 0.282 & 0.381 & SW \\
\hline Fe XIII $209.9(\mathrm{bl}) / 202.0$ & $0.15(10 \%)$ & & $0.025(0.005,1.9 / 76.8)$ & 0.169 & 0.159 & BR SW \\
\hline Fe XIII 204.9 (bl)/201.1 (bl) & $0.31(30 \%)$ & $0.27(-13 \%)$ & $0.088(0.01,2.2 / 24.7)$ & 0.289 & 0.278 & BR SW \\
\hline Fe XIII $201.1(\mathrm{bl}) / 197.4$ & $5.0(30 \%)$ & $6.2(+24 \%)$ & $2.65(0.5,24.8 / 9.3)$ & 0.540 & 0.416 & BR SW \\
\hline Fe XIII 204.9 (bl)/197.4 & $1.55(30 \%)$ & $1.7(+10 \%)$ & $0.23(0.04,2.2 / 9.3)$ & 0.154 & 0.115 & BR SW \\
\hline Fe XIII 200.0/196.5 (bl) & $3.17-3.28(3.3,20 \%)$ & $3.3(0 \%)$ & $1.66(0.3,13.1 / 7.8)$ & 0.512 & 0.576 & SW \\
\hline Fe XIII 209.6/200. & $0.74-0.685(0.68,20 \%)$ & & $0.05(0.01,1.7 / 32.4)$ & 0.072 & 0.081 & SW \\
\hline Fe XIII 246.2/251.9 & $0.51(10 \%)$ & $0.59(17 \%)$ & $0.34(0.01,2.9 / 8.4)$ & 0.651 & 0.635 & BR LW \\
\hline Fe XIII 251.9/201.1 (bl) & $1.3(20 \%)$ & & & & & LW/SW \\
\hline Fe XIII 251.9/204.9 & $4.2-4.8$ & & $2.73(42.6 / 15.6)$ & 0.700 & 1.164 & LW/SW \\
\hline Fe XIV 252.2/264.8 & $0.23(10 \%)$ & 0.25 & $0.08(0.01,3.7 / 44.5)$ & 0.331 & 0.421 & BR LW \\
\hline Fe XIV $257.4 / 270.5$ & $0.68(10 \%)$ & & $0.32(0.02,7.9 / 25.7)$ & 0.448 & 0.541 & BR LW \\
\hline Fe XIV 289.1/274.2 & $0.065(20 \%)$ & $0.065(0 \%)$ & $0.013(0.002,0.6 / 47.7)$ & 0.211 & 0.232 & BR LW \\
\hline Fe XIV 274.2/211.3 & $0.69(10 \%)$ & $0.77(10 \%)$ & $4.9(0.4,233 / 47.5)$ & 9.225 & 9.636 & $\mathrm{LW} / \mathrm{SW}$ \\
\hline Fe XIV 270.5/(264.7+274.2) & $0.26(20 \%)$ & $0.26(0 \%)$ & & & & LW \\
\hline Fe XVI 251/263 & $0.57(20 \%)$ & $0.66(16 \%)$ & $0.23(0.02,3.9 / 16.6)$ & 0.388 & 0.408 & LW \\
\hline Fe XVII 254.9/204.7 & $1 / 0.93(10 \%)$ & & $0.64(0.06,5.6 / 8.7)$ & 0.744 & 1.448 & BR LW/SW \\
\hline Fe XXIV 255.1/192 & $1 / 1.85(10 \%)$ & & $0.067(0.007,3.5 / 52)$ & 0.166 & 0.207 & LW/SW \\
\hline Si VII 275.7/272.6 & $0.58(10 \%)$ & & $0.46(0.07,0.1 / 0.3)$ & 0.805 & 0.865 & BR LW \\
\hline Si VII $275.4 / 272.6$ & $4.2-3.45$ & & & & & LW \\
\hline Si $\mathrm{X} 253.8 / 258.4$ & $0.19(10 \%)$ & $0.18(5 \%)$ & $0.13(0.01,0.2 / 1.3)$ & 0.658 & 0.709 & BR LW \\
\hline Si X 277.2/272. & $0.84(10 \%)$ & $0.87(2 \%)$ & $0.53(0.02,0.6 / 1.2)$ & 0.646 & 0.758 & BR LW \\
\hline Si $x$ 261.1/277.2 & $1.37-1.35(10 \%)$ & $1.36(0 \%)$ & $1.41(0.08,0.9 / 0.6)$ & 0.969 & 0.926 & LW \\
\hline $\mathrm{S} \times 257.1(\mathrm{bl}) / 264.2$ & $0.348(10 \%)$ & & $0.24(0.03,0.2 / 0.9)$ & & & BR LW \\
\hline S XI $285.6(\mathrm{sbl}) / 281.4$ & $0.496(10 \%)$ & & $0.31(0.05,0.3 / 1.1)$ & 0.634 & 0.635 & BR LW \\
\hline Si VII 275.4/Fe VIII 185.2 & & 0.63 & $0.61(0.06,2.6 / 4.3)$ & 1.432 & 1.217 & LW/SW \\
\hline
\end{tabular}

Notes. The second column shows the theoretical ratios (photon units) within the log $\mathrm{Ne}\left[\mathrm{cm}^{-3}\right]=8-9$ range, and optionally the chosen value with an estimated uncertainty. The third column indicates the observed ratios from Malinovsky \& Heroux (1973), and in brackets the percentage difference with the predicted ratios. The following column indicates the EIS averaged ratio (in data numbers per second, DN/s), with its variation in brackets (1 sigma) and the averaged values of the two lines (in DN/s). The following two columns show the ratios of the effective areas $R_{\text {eff }}$ obtained with the present calibration and the ground calibration. The last column shows the channel, and whether the ratio is a branching ratio (BR).

A summary of the main line ratios chosen for the calibration is given in Table 1. A differential emission measure (DEM) analysis (see Del Zanna 1999 for the method used) on many averaged spectra (on-disk, off-limb, over the quiet Sun, and active regions) has been performed, to assess possible known sources of blending. Only the relevant findings are summarised below.

\subsection{Fe VIII}

We have performed a preliminary assessment of the Fe VIII lines in terms of their use for calibration purposes. We used the atomic data (and identifications) for Fe VIII given in Del Zanna (2009b), and compared them with the recent large-scale atomic calculation for this ion performed by Tayal \& Zatsarinny (2011). Most predicted line intensities are very similar, within $10 \%$. However, for a few lines larger discrepancies (30-50\%) were found. These discrepancies originate from differences in the atomic structure calculations of Tayal \& Zatsarinny (2011) and Del Zanna (2009b), which reflect in different oscillator strengths, as shown in Tayal \& Zatsarinny (2011). The affected lines originate from highly mixed levels, which include several strong lines observed by EIS, and were the focus of the Del Zanna (2009b) study. They are the 185.2, 186.6, 194.6, and $197.3 \AA$ lines. The large-scale Del Zanna (2009b) structure calculations provide energies in closer agreement to the experimental values, and provide oscillator strengths in close agreement with the large-scale calculations of Zeng et al. (2003). It therefore appears that the Tayal \& Zatsarinny (2011) calculations are not accurate for these lines. However, for the $196.0 \AA$ line good agreement in the oscillator strengths is found. The 185.2/196.0 $\AA$ ratio is one of the most reliable. We adopt a value of 5.3 photons at $\log \mathrm{Ne}\left[\mathrm{cm}^{-3}\right]=9$ and $\log \mathrm{Te}[\mathrm{K}]=5.65$, obtained with the Del Zanna (2009b) data, distributed within CHIANTI. 


\subsection{FeIX}

A few Fe IX lines from the $3 \mathrm{~s}^{2} 3 \mathrm{p}^{4} 3 \mathrm{~d}^{2}$ configuration, identified by Young (2009), could be used for the calibration, although with various limitations. No branching ratios are available, and the lines are close in wavelength. Many of the lines were found blended during the present assessment. For example, the $188.4 \AA$ with Mn IX, the 189.6 $\AA$ with Ar XI, and the 191.2 $\AA$ line with $\mathrm{S}$ XI. These blends are all difficult to estimate. We are left with the 189.9 and the $197.9 \AA$ lines. The ratio of these lines is predicted to vary with both density and temperature by a significant amount, about $30 \%$ in the temperature interval where Fe IX is expected to be formed, and $20 \%$ in the $\log \mathrm{Ne}\left[\mathrm{cm}^{-3}\right]=8-9$. A value of 1 is assumed.

There is relatively good agreement between predicted (Storey et al. 2002) and observed (using the ground calibration) intensities for the EIS Fe IX lines (Young 2009; Del Zanna 2009a); however, the predicted intensities for these lines are quite uncertain. The new scattering calculations for the other iron ions mentioned in the introduction have shown that cascading and resonance excitation due to the larger targets can affect both high- and low-energy levels by changing the populations up to $30-40 \%$. Work has started on a similar large-scale calculation for Fe IX to improve the current atomic data.

There is also the resonance $171 \AA$ line, but it is barely visible in the EIS spectra where the EIS sensitivity is about three orders of magnitude lower than at the peak around $195 \AA$. This line is also strongly density- and temperature-sensitive, as shown in Young (2009) and Del Zanna (2009a).

\subsection{FeX}

The FeX identifications are summarised in Del Zanna et al. (2004). The recent atomic calculations of Del Zanna et al. (2012b) are adopted here. The new data significantly affect the 257.26 ̊ self-blend, the strongest Fe X line in the LW channel (identified in Del Zanna et al. 2004). One problem is that the ratio of this self-blend with the lines in the SW band is both slightly density and temperature sensitive, and is not ideal for checking the SW/LW relative calibration.

The $174.5,177.2$, and $184.5 \AA$ lines are all well observed in the SW channel. Their ratios do not depend on density or temperature, and excellent agreement (to within a few percentage points) with the MH73 observations is found. The QS observations have been selected for these lines. The $184.5 / 190 \AA$ is in principle an excellent branching ratio, with both lines well observed. The $190 \AA$ line is blended with at least a known Fe XII line (see below). Further blending cannot be excluded, although the Fe $\mathrm{X} 184.5$ vs. $190.0 \AA$ deblended branching ratio shows a small scatter. The ratio has therefore been assigned a larger uncertainty $(30 \%)$.

The 207.4/184.5 $\AA$ has a predicted ratio of about 0.15 photons using the Del Zanna et al. (2012b) data. We note that there is a significant enhancement (factor of two) of the $207.4 \AA$ line, compared to the previous atomic calculations of Del Zanna et al. (2004), due to the larger scattering calculation. On the other hand, the new calculations over-predict the $193.7 \AA$ line, which turns out to be very sensitive to the target employed, so it is not reliable for the calibration.

\subsection{FeXI}

Fe XI produces a number of strong EIS lines. New identifications of many of them were presented in Del Zanna (2010), using the atomic calculations of Del Zanna et al. (2010b). A few temperature-sensitive lines around $255 \AA$ were identified, in particular the self-blend at $257.5 \AA$. Excellent agreement with the MH73 data was found for a few Fe XI lines (Del Zanna 2010), providing confidence in the calculations that resolved large (factors of 2-3) discrepancies. Here, the recent large-scale atomic calculations of Del Zanna \& Storey (2013) are adopted. They have improved the atomic data for the lines around $255 \AA$. For example, Table 4 in Del Zanna \& Storey (2013) shows that the intensity of the $257.5 \AA$ line increases by $46 \%$. Such large increases were required to reduce the large disagreements between predicted observed intensities in the EIS lines around $255 \AA$; these EIS lines had intensities much weaker than predicted, as discussed in (Del Zanna 2010). Further discrepancies were due to the use of the ground calibration, as discussed below.

There are three good branching ratios in the SW channel. The 188.2/192.8 $\AA$ ratio is complex, as discussed at length in Del Zanna (2010). The 188.2 $\AA$ line is mainly due to Fe XI, while the $192.8 \AA$ line is blended with a host of O V lines, and in active regions with CaXVII, as described in Young et al. (2007). The benchmark of a clean transition-region (TR) spectrum has shown good agreement between the $192.8 \AA$ and the $192.9 \AA$ O V lines, indicating that no further blending at $192.8 \AA$ with a cool line is present, as shown in Del Zanna (2009a). When off-limb observations (to avoid the presence of $\mathrm{OV}$ lines) are considered, there is a significant and puzzling disagreement in this Fe XI branching ratio (when the ground calibration is used). One explanation would be the presence of a strong unidentified coronal line, contributing about $30 \%$ to the intensity of the observed line (Del Zanna 2010). There is a further Fe XI line at $192.8 \AA$, with a well-established branching ratio with the $188.3 \AA$ (Del Zanna et al. 2010b), but this line is very weak. The deblended QS ondisk 188.2/192.8 $\AA$ ratio is remarkably constant in time, with a value of $2.0 \pm 0.1$. If a blending with a coronal line were present, the unidentified line would have to have the same temperature of formation of Fe XI, an unlikely coincidence. Considering the other calibration ratios, we assume here that there is no unidentified line. We adopt a QS off-limb value of 2.1 for this ratio.

The Fe XI 202.7/188.3 ^ branching ratio is more uncertain than other ratios, given the large range of values that are obtained with different atomic structure calculations. The target adopted for the Del Zanna et al. (2010b) scattering calculation overestimates the $202.7 \AA$ line by about $30 \%$, according to the largest structure calculation (48CT), as shown in the same paper. We adopt here a branching ratio of 0.1 , from the $48 \mathrm{CT}$ calculation. The third branching ratio, $178.1 / 182.2 \AA$, is only measurable in active region (AR) observations, because of low signal in the first line.

The 180.4/188.2 $\AA$ ratio is well established in terms of atomic calculations (Del Zanna et al. 2010b). A conservative $20 \%$ uncertainty is given to this ratio. The 1-35 201.11 $\AA$ transition (Del Zanna 2010) has increased excitation in the latest calculation, increasing its emissivity by $45 \%$. A ratio of 0.032 (photons) with the strongest $180.4 \AA$ line was adopted to deblend the Fe XIII 201.1 $\AA$ line. The Fe XI 257.5/188.2 is a good crosschannel ratio, although it is slightly temperature-dependent. It varies by $14 \%$ in the $\log T[K]=6.0-6.1$ range.

\subsection{FeXII}

Several new identifications of important EIS Fe XII lines were presented in Del Zanna \& Mason (2005), in particular the 
self-blends at 186.9 and $195.1 \AA$ used extensively to measure densities. Here, we use the large-scale atomic calculations of Del Zanna et al. (2012a) where we obtained significant improvements for the 186.9 and $196.5 \AA$ lines over our previous calculations (Storey et al. 2005). The intensities of these lines are about $30 \%$ higher, providing lower electron densities, now in agreement with those obtained from other ions.

The $192.4,193.5$, and $195.1 \AA$ lines are extremely strong in any observation and their ratios are not sensitive to density and temperature (at high densities the $195.1 \AA$ does change), so they are excellent for the calibration. The MH73 observations are in exact agreement with theory. A large quantity of dust on the EIS CCD affects the $193.5 \AA$ line, so the area was avoided.

The $186.9 / 196.6 \AA$ ratio is only slightly dependent on density and is used here. We note that the $196.6 \AA$ line is slightly blended with a weak Fe VIII transition (Del Zanna 2009a). The QS observations were used for the calibration. In principle, the 291/186.2 A ratio could be a good calibration ratio, but the lines are weak and only measurable in AR spectra. The $291 \AA$ measurement is very uncertain because the line is at the very edge of the LW channel. The weak $186.2 \AA$ line appears to be severely blended, not just with a known Fe XI line.

There is a $190.07 \AA$ line blending Fe X (see above). Its intensity has been estimated from the observed $192.4 \AA$ line, assuming a ratio of 0.023 photons. This ratio, with the Del Zanna et al. (2012a) atomic data, ranges from 0.02 to 0.028 in the log $\mathrm{Ne}\left[\mathrm{cm}^{-3}\right]=8-9$ range.

Del Zanna \& Mason (2005) gave a tentative suggestion for the identification of the strongest decay from the $3 \mathrm{~s} 3 \mathrm{p}^{3} 3 \mathrm{~d}$ configuration (from the ${ }^{4} \mathrm{D}_{7 / 2}$ 6-84 line), as a blend with another Fe XII line observed at $191.05 \AA$. However, Hinode/EIS spectra showed the 191.05 A line to be too weak (Del Zanna 2012). The energy difference between observed and theoretical values for the lower $3 \mathrm{~s}^{2} 3 \mathrm{p}^{2} 3 \mathrm{~d}$ configuration suggests a wavelength around $188 \AA$. There are a few possibilities, in particular that the line is blended with the strong Fe XI 188.2 and $188.3 \AA$ lines, or is the previously unidentified coronal line observed by Del Zanna (2012) at $188.37 \AA$, which has about the right intensity. Del Zanna (2012) suggested that this line could be blended with the Fe X $190.0 \AA$ line. However, the Fe X 184.5 vs. $190.0 \AA$ branching ratio is very constant in time with a small scatter, which suggests that no significant blending of the $190.0 \AA$ line is present. If the Fe XII 6-84 line intensity is deblended from the $190 \AA$ line, the Fe X 184.5/190.0 $\AA$ ratio would increase over time instead of being constant at it should be.

There is a weak 201.1 $\AA$ line (Del Zanna \& Mason 2005) blending the strong Fe XIII transition. The intensity of the Fe XII line has been estimated from the observed 191.05 line assuming a ratio of 0.24 (photons). There are other Fe XII lines, but they are either weak, strongly density-sensitive, or show significant disagreement with theory. For example, it has been suggested (Young, priv. comm.) that the $249.3 \AA$ line could be useful. This line was identified in Del Zanna \& Mason (2005) as Fe XII; however, the observed intensity is significantly stronger than predicted. The DEM modelling for the QS indicates a discrepancy of a factor of two, so this line is either blended, the identification is incorrect, or the excitation rates for this line are incorrect.

\subsection{Fe XIII}

There are several Fe XIII lines useful for the calibration. The new identifications presented in Del Zanna (2011) are adopted here, together with the atomic calculations of Storey \& Zeippen (2010); Del Zanna \& Storey (2012). The Fe XIII lines are strong in off-limb QS and AR spectra, so only these observations have been considered. The 246.2 and $251.9 \AA$ lines form a branching ratio, predicted at 0.51 (photons), in excellent agreement with MH73 (0.59). The observed intensities of the 246.2 and $251.9 \AA$ lines were found to be too low by a factor of at least 1.5 (Del Zanna 2012), while they are in excellent agreement when the MH73 spectrum is considered.

The branching ratio 209.9/202.0 $\AA$ is also good, although the $209.9 \AA$ line is slightly blended with an unidentified TR line (Del Zanna 2009a). In on-disk AR observation, the average ratio in $\mathrm{DN}$ is 0.03 . We adopt the off-limb 2007 Aug 19 value of 0.025 for this ratio. The $200.0 / 196.5 \AA$ ratio is not very dependent on density, so it is also used. We note that the $196.5 \AA$ line is blended in on-disk observations with an unidentified TR line (Del Zanna 2009a). We use an off-limb observation for the $200.0 / 196.5 \AA$ ratio.

The 251.9/201 $\AA$ ratio varies only slightly with density and offers in principle a way to cross-calibrate the SW and LW channels. However, as pointed out in Del Zanna (2011), the atomic calculations for the 197.4, 201.1, and $204.9 \AA$ lines, all originating from the same upper level, provide branching ratios that are somewhat more uncertain than in other cases.

The $197.4 \AA$ line is relatively weak and has other lines nearby, so extreme care is needed to measure it. The 201.1 transition is considerably blended (up to $15 \%$ in AR spectra) with two known transitions from Fe XI and Fe XII, described in the relative sections. The 201.1/197.4 $\AA$ branching ratio is lower in off-limb AR spectra, an indication of further blending with a lower-temperature line. The averaged ratio in AR on-disk observations is $3.1 \pm 0.5$, while in the off-limb 2007 Aug 19 observation it was 2.65 , a value adopted here.

The 201.1/197.4 $\AA$ branching ratio has a theoretical value of 5.0 using the Del Zanna (2011) data. In the MH73 spectrum the observed ratio was 6.2 , in quite good agreement once the blending in the $201.1 \AA$ line is taken into account. The $204.9 / 197.4 \AA$ theoretical branching ratio is 1.55 , close to the MH73 value of 1.7. We note that the $204.9 \AA$ line is blended with a weak TR line, so the off-limb 2007 Aug 19 observation is adopted here.

\subsection{FeXIV}

Lines from Fe XIV are of particular importance for the calibration of the LW channel and the cross-calibration between the SW and LW. Significant discrepancies in the LW line ratios and in the LW/SW ratio were found (Del Zanna 2012). Only AR observations are used here. In the QS, most of the Fe XIV lines are hardly visible and become severely blended. For example, a new Fe XI transition was identified by Del Zanna (2010) as blended with the $264.7 \AA$ line.

We use the atomic data of Storey et al. (2000). Liang et al. (2010) performed a larger scattering calculation and found very similar ratios (within a few percentage points). The only exception was the $289.1 / 274.2 \AA$ branching ratio, for which Liang et al. (2010) has 0.08 photons, and Storey et al. (2000) has 0.065 photons. This ratio is sensitive to the target employed. The latter value is adopted here, because it agrees with the 
MH73 value, obtained by deblending the $274.2 \AA$ line. We note that the $274.2 \AA$ line is blended with a Si VII transition that has a strong density sensitivity. The intensity of this line has been roughly estimated here as 0.2 times the intensity of the $275.3 \AA$ line.

The branching ratios 252.2/264.8 $\AA$ and 289.1/274.2 show excellent agreement with the MH73 spectrum. The Fe XIV $257.4 / 270.5 \AA$ branching ratio is predicted to be 0.68 , but is often larger, a possible indication of a blend in the weaker $257.4 \AA$ line. The ratio of the $270.5 /(264.7+274.2)$ lines is predicted to be 0.26 photons, exactly as observed by MH73. The 274.2/211.3 $\AA$ ratio does not depend strongly on density and temperature. Good agreement (within a relative 10\%) is found with the MH73 data.

\subsection{FeXVI, FeXVII, and FeXXIV}

Two strong lines are produced in active regions by Fe XVI; they are not density-sensitive and offer, in principle, an excellent calibration ratio. There are only three lines at the EIS wavelengths, the strongest of which is the $262.98 \AA$ line. We use the APAP atomic data of Liang et al. (2009b). The Fe XVI $265 \AA$ line appears to be blended, while the $251 / 263 \AA$ predicted ratio is not in very good agreement with the MH73 observations.

Del Zanna \& Ishikawa (2009) have identified/benchmarked Fe XVII lines in the EIS channels. Here, the atomic data of Liang \& Badnell (2010) are used. The two main lines for this ion, at 204.7 and $254.9 \AA$, form a branching ratio. As for the Fe XXIV 192.0/255.1 $\AA$ ratio, significant discrepancies were found by Del Zanna (2008) and Del Zanna \& Ishikawa (2009), with the LW lines being much weaker than expected. The Fe XVII $204.7 \AA$ line was found by Del Zanna \& Ishikawa (2009) to be blended with a low-temperature line, identified as partly due to Fe VIII (Del Zanna 2009b). However, the blending did not resolve the issue. Opacity effects would make the problem worse, so the only explanation is a problem in the EIS calibration.

Flare observations of the right type are needed to use Fe XVII and Fe XXIV lines for the calibration. Lines from these ions are blended in small flares, as discussed in Del Zanna et al. (2011). In large flares (M-class), the $192.0 \AA$ line is normally saturated in the EIS observations. The Fe XXIV lines are strong during the impulsive phase of flares, but often exhibit strong blue-shifted components which complicate the analysis.

\subsection{Silicon and sulfur lines}

Six lines are of particular importance for the calibration of the LW channel since they are among the strongest lines in quiet conditions, and there are three good ratios to use. We use the APAP atomic data of Liang et al. (2009a) and the QS observations.

The Si VII 275.7/272.6 $\AA$ is a good branching ratio. We use QS observations. There are no Si VII lines in the SW channel, but Kamio \& Mariska (2012) and Mariska (2013) suggested the use of the Si VII 275.3 $\AA$ vs. Fe VIII 185.2 A ratio to monitor the relative sensitivity of the LW/SW channels. We will discuss this issue below.

The Sx 257.1/264.2 ratio should, in principle, be a good ratio (0.348 photons), but the weak $257.1 \AA$ line often appears blended, especially in AR spectra, where at least the Fe XIII $257.17 \AA$ line produces a contribution. We use
QS observations, where good agreement with the ground calibration is found. The S XI 285.6/281.4 $\AA$ is a good branching ratio in active region spectra. The S VIII 198.55 $\AA$ line is one of the few low-temperature lines in the SW channel. In quiet Sun regions this line is blended by about $30 \%$ with Fe XI, according to $D E M$ modelling.

\subsection{Data selection and processing}

The above discussion has made clear that a variety of targets were needed for the calibration. There are surprisingly few good observations suitable for calibration purposes. Very few EIS studies telemetred the full spectral range. Most observations in the first few years were done with the 1 "slit and very low counts in the QS are present. Upon my suggestion, spectral atlases with the $2^{\prime \prime}$ slit (and long exposures) have been run since 2010 for monitoring purposes (Atlas_30, Atlas_60, Atlas_120). They have been extremely useful for the present work and should be continued.

A selection of QS data was chosen by inspecting the spectra and avoiding observations with strong hot lines. This was relatively simple in the first three years of the mission, but since then very few QS observations have been made. One further complication is that the typical QS disappears at temperatures above 1 MK (Del Zanna \& Andretta 2011) when solar activity increases. A variety of EIS studies were selected. A selection of AR observations was also chosen, to use the ratios involving the Fe XII-Fe XVI ions (avoiding saturated areas). A full list is given in the Appendix.

A few particular observations are worth mentioning. To study the EIS sensitivity during the first month of routine operations (to limit instrument degradation), two dates were chosen. A QS observation on 2006 Dec. 23 was selected with two regions, one on-disk and one off-limb. An AR on-disk observation of hot core loops on 2006 Dec. 25 was also selected, to build ratios of the hotter lines.

For the flare lines, a suitable observation was searched for but was not found. The search criteria were to find any EIS flare observation within the first eight months of at least a C-class flare and which contained the two Fe XVII and Fe XXIV line ratios. So, the observations of 2007 Jun. 2 discussed in Del Zanna (2008) are considered here.

To increase the signal-to-noise, observations with long exposures have been selected. These have the drawback of increasing the incidence of cosmic rays. The Solarsoft routine new_spike (written by Young) was used to automatically flag the cosmic ray hits as missing data. The so-called hot and warm pixels as listed within the EIS software were also flagged as missing data. Each exposure was then visually inspected with custom-written software. This was necessary because occasionally the automatic routine does not detect cosmic rays. It was also necessary to note data dropouts or regions with high particle fluxes, a common feature in EIS observations.

The number of hot and warm pixels in the EIS CCD has increased so much that since 2009 all observations have needed special processing. The missing data were replaced with interpolated data, obtained by successive interpolation along and across the slit direction, and visual inspection/manual corrections of each exposure. This interpolation was necessary before spatial averaging and line fitting.

Various geometrical corrections have been applied to the data (see Del Zanna 2009b, for details). A slant in the spectra was found in Del Zanna \& Ishikawa (2009). This results in a 
wavelength dependent offset along the slit and has been corrected for by rotating each exposure and aligning the SW and LW channels. The offset of about $2^{\prime \prime}$ in the pointing of the SW and LW channels prior to 2008 Aug. 24 was also taken into account.

Significant spatial averaging was needed to measure the weaker lines, given the low signal in most exposures. Single-slit exposures were averaged along selected regions along the slit (typically over more than 100"), while raster observations were first analysed to select the best regions to obtain averaged spectra. QS regions were selected as those where no bright points or any strong hot (above $2 \mathrm{MK}$ ) emission was present. On the contrary, AR spectra were obtained from regions within the cores, where the Fe XIV and Fe XVI lines, needed for the calibration, were strong. Regions where the strongest lines are saturated were avoided. Off-limb regions were selected by avoiding any particular structures.

Each of the resulting spectra was wavelength calibrated (the EIS wavelength scale varies along the orbit). All the EIS lines were fitted with Gaussian profiles using the $c$ fit package (Haugan 1997), directly on the raw spectra in data numbers (DN). One distinct feature of the EIS spectra is a variable bias. In particular, the SW channel shows an enhanced background in the center, which we believe to be mostly due to a pseudo-continuum of spectral lines. The way the background is chosen affects the spectral fitting of the weak lines. Typical uncertainties in the lines are small, of the order of a few percent for the strong lines. Uncertainties were estimated by summing in quadrature the photon noise in the line and in the background, and by taking into account the spatial averaging. All the fits were checked visually.

From the total number of counts in a line $N(\mathrm{DN})$, the calibrated observed intensity $I_{\mathrm{o}}\left(\right.$ phot cm $\mathrm{cm}^{-2} \mathrm{~s}^{-1} \operatorname{arcsec}^{-2}$ ) is obtained by (see EIS software note No. 2 for details)

$$
I_{\mathrm{o}}=\frac{3.65 N \lambda G}{12398.5 \Omega E(\lambda) t}
$$

where $\lambda$ it the wavelength in $\AA, t$ the exposure time in seconds, $\Omega$ is the solid angle subtended by each pixel in arcseconds square ( 1 for the $1^{\prime \prime}$ slit, 2 for the $2^{\prime \prime}$ slit), 12398.5 a conversion factor, $G$ is the gain of the CCDs, assumed to be 6.3, 3.65 the number of $\mathrm{eV}$ to produce an electron-hole in the CCD (silicon-based), and $E(\lambda)$ is the effective area, i.e. the area of the aperture multiplied by the various geometrical factors and the transmission coefficients of the optical parts of the instrument (the transmission of the filters, the mirror reflectivity, the grating reflectivity, etc.).

The ground end-to-end calibration at RAL (Lang et al. 2006) only provided measurements at one wavelength for the SW (205.9 A) and four wavelengths for the LW (251.3, 256.3, 267.25, 283.4 $\AA$ ). These measurements were compared to those predicted by combining all the efficiencies of the various optical components, which were also measured in the laboratory. Significant discrepancies between the measured and predicted responsivities were reported by Lang et al. (2006), both in terms of absolute values and in terms of relative values within each channel. The disagreement in the absolute values was not surprising, considering the large uncertainty in the quantum efficiency of the detectors. Scaling the predicted values (by a factor of 1.6) however, still left a discrepancy of about $20 \%$ between the SW and the LW channel, and about $50 \%$ within the four measurements in the LW channel. Lang et al. (2006) suggested the use of new effective areas, obtained by combining the five calibration points with the predicted shapes of the responsivities. An overall uncertainty of $22 \%$ was estimated. These effective
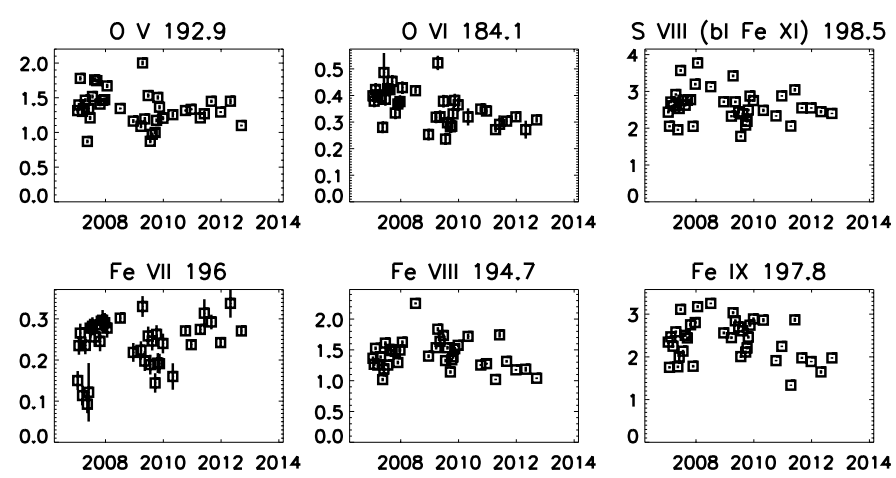

Fe IX 197.8
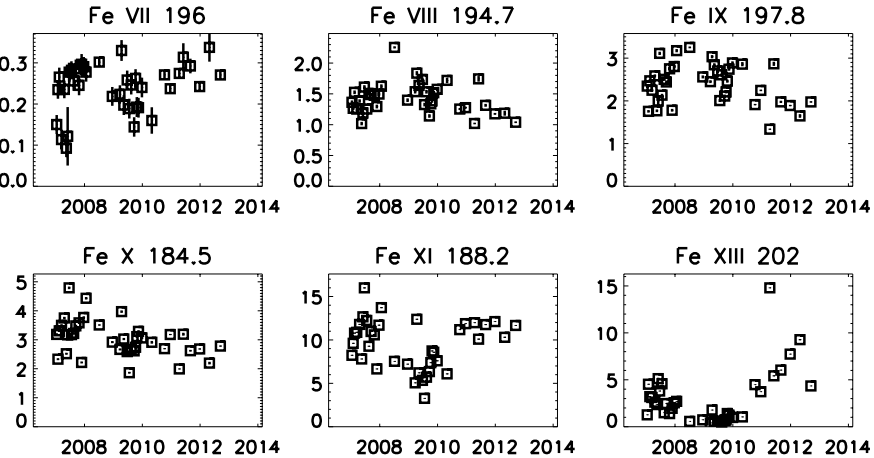

He II (dbI) 256.3
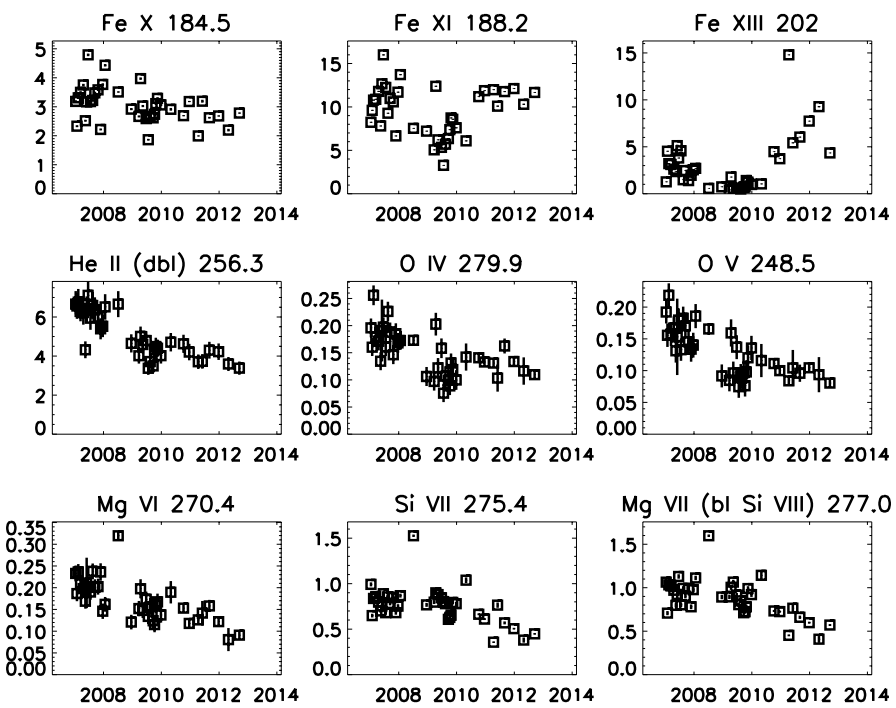

Fig. 1. Averaged EIS count rates (DN/s per $\left.1^{\prime \prime}\right)$ in the QS areas as a function of time. The He II $256.3 \AA$ line has been deblended.

areas are available via SolarSoft (files EIS_EffArea_A.004 and EIS_EffArea_B.004).

\section{Results}

\section{1. $S W$ and $L W$ line radiances}

We first present the averaged raw count rates in the QS areas in Fig. 1. The large scatter is mainly due to solar variability, however the cooler TR lines in the SW channel do show a remarkable constancy over time, indicative of no significant long-term degradation. The main TR line is the strong $\mathrm{O} v$ self-blend, close to the peak of the SW effective area, but other strong TR lines from S VI, Fe VII, Fe VIII, and Fe IX, all show the same trends. However, lines short-ward of $186 \AA$ (such as O VI $184.1 \AA$, shown in the figure) do show a decrease, indicative of a small degradation at the shorter wavelengths. This will be discussed below.

The constancy of the TR line radiances and the direct EUNIS/EIS measurements of Wang et al. (2011) suggest that the absolute SW central responsivity has not decreased significantly over time. This is assumed in the remainder of the paper. We note that this result is in stark contrast to the conclusions reached by Kamio \& Mariska (2012) and Mariska (2013). We will return to this point in the conclusions.

All the cool lines in the LW channel do, however, show a decrease in their QS radiances, as also shown in Fig. 1. The He II $256.3 \AA$ line (which is actually a self-blend) is the strongest 

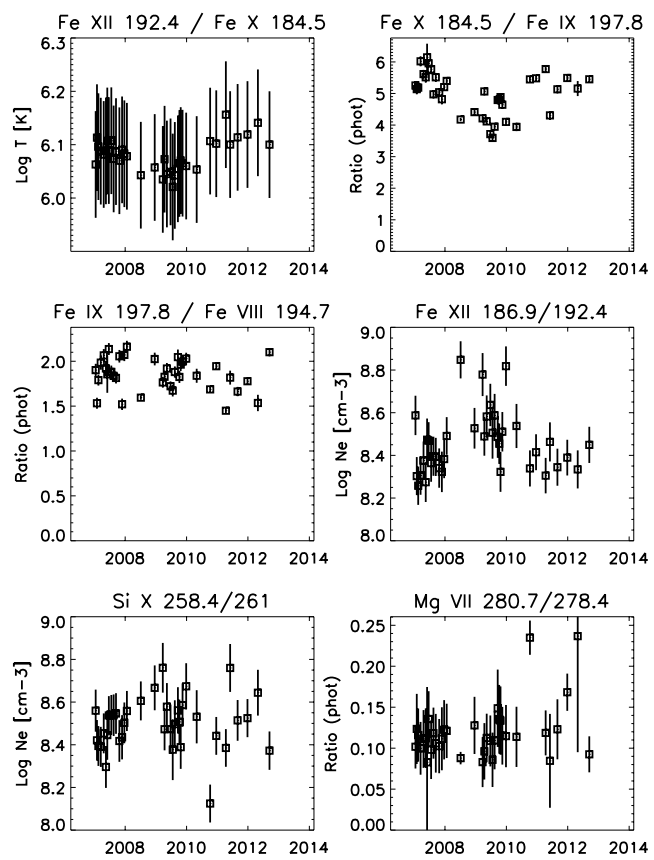

Fig. 2. Ratios of a few lines (photon units, using the ground calibration), with densities and temperatures obtained from them.

line in the channel and shows a marked decrease, by about a factor of two within the first two years. The He II $256.3 \AA$ line is blended with several transitions on its long wavelength side, mainly from Si X, Fe X (Del Zanna et al. 2004), Fe XII (Del Zanna \& Mason 2005), and Fe XIII (Del Zanna 2011). Careful deblending of off-limb observations indicates the possible presence of further minor blending.

Estimating the blends is not simple, as the lines are densitysensitive. Fortunately, the main contribution is from the SiX $256.4 \AA$ line, forming a branching ratio with the Si X $261 \AA$ line, which can be estimated quite accurately. The broad profile of the He II blend was fitted with two Gaussians, leaving all parameters to vary. The total intensity in the blend was then obtained by summing the two intensities. The contributions from the other ions was then subtracted, using the measured (calibrated) intensities in the Si X 261, Fe X 257.2, Fe XII 192.4, and Fe XIII $252.0 \AA$ lines. The result is that, in on-disk QS observations, the He II line dominates by contributing over $80 \%$ to the observed intensity. A similar result was found by Mariska (2013), where however the He II $256.3 \AA$ line intensity was simply estimated by a double Gaussian fit.

As pointed out by Kamio \& Mariska (2012) and Mariska (2013), significant solar cycle changes are present in lines formed above $1 \mathrm{MK}$, (such as Fe XIII), as expected (Del Zanna et al. 2010a; Del Zanna \& Andretta 2011). Before proceeding further, it is then important to estimate how the solar cycle variations can affect densities and temperatures in the QS observations considered here. This is shown in Fig. 2. The count rates were converted into calibrated radiances (photon units) using the ground calibration.

An estimate of temperature changes was obtained from the Fe XII 192.4 $\AA / F$ X $184.5 \AA$ ratio, using the CHIANTI v.6 ionisation equilibrium. Small changes within $\log T[\mathrm{~K}]=6-6.2$ are present, with decreasing temperatures toward the minimum on 2008, and an increase. The Fe X/Fe IX ratios also show a similar behaviour, which is simply because lines formed above $1 \mathrm{MK}$ increase their intensities. Instead, the Fe IX/Fe VIII ratios indicate very little change in the upper QS TR temperature. Densities have also been measured from two among the best EIS ratios, one from Fe XII and one from Si X, using lines close in wavelength to reduce degradation effects. The densities of the two ions agree very well (with the new atomic data for both ions), and show little variability around $\log \mathrm{Ne}\left[\mathrm{cm}^{-3}\right]=8.4$. The Mg VII ratio (see Del Zanna 2009a, for details on blending issues) also indicates no significant changes in the density of the QS upper transition region.

\subsection{Calibrated radiances}

One question naturally arises: how do the calibrated radiances compare with previously well-calibrated measurements for the stronger lines? As discussed in Del Zanna et al. (2010a); Del Zanna \& Andretta (2011) lines formed in the transition region up to $1 \mathrm{MK}$ have been found to have irradiances that are not significantly affected by solar activity (presence of active regions), and are also expected to have radiances in quiet Sun regions that are approximately constant. So although solar variability is always present, by spatially averaging over large regions one should obtain EIS radiances in agreement with other well-calibrated measurements.

As discussed in Del Zanna \& Andretta (2011), the best calibrated irradiances in the EUV are those obtained by Malinovsky \& Heroux (1973), Heroux et al. (1974; hereafter H74), together with Manson (1976; hereafter M76), and those we obtained from PEVE, the prototype of the Solar Dynamics Observatory (SDO) Extreme ultraviolet Variability Experiment (EVE), flown on 2008 April 14 during the deep solar minimum (Woods et al. 2009; Chamberlin et al. 2009). These values are shown in Table 2. The He II 256.3 $\AA$ line has been deblended from the strong Si X contribution using the other Si X lines and theory, for both MH73 and H74 data, although in principle MH73 lists $5.2 \times 10^{8}$ phot $\mathrm{cm}^{-2} \mathrm{~s}^{-1}$ as already deblended. The fact that good agreement exists between MH73 and H74 for the other He II 237 and $243 \AA$ lines (see Table 2) suggests a mistake in the MH73 list. The PEVE measurement of the $256.3 \AA$ line is quite uncertain given the low resolution of the instrument.

It is well known that it is possible to obtain an estimate of the QS radiances at disk center from the irradiances, once a correction factor for the limb-brightening is known. It is also well known that helium lines do not show any limbbrightening, while all the TR lines do. We have recently measured (Andretta \& Del Zanna 2013) these limb-brightenings using the SOHO/CDS radiances for a range of ions, and found correction factors close to 1.4 for all lines formed in the lower and upper TR, up to $1 \mathrm{MK}$. We also estimated the correction due to the off-limb contribution (Del Zanna et al. 2010a), but this is negligible (a few percentage points at most) for these lines. We have therefore applied a correction factor of 1.4 to obtain estimated QS radiances from the irradiance measurements. These QS radiances are also shown in Table 2 in brackets. In terms of actual QS radiances, very few observations at the EIS wavelengths exist. There are the SOHO GIS observations (e.g. Del Zanna 1999; Andretta et al. 2003), although the GIS lines were calibrated with the line ratio technique (Del Zanna et al. 2001). It is interesting to note that good agreement is found between these GIS measurements and the EIS measurements. The SERTS-95 rocket flight also measured QS radiances for a few of the brightest lines, but older CHIANTI atomic data were used for its calibration, using the line ratio technique (Brosius et al. 1998) 
Table 2. Quiet Sun historical irradiances and radiances.

\begin{tabular}{lcccccc}
\hline \hline & $\log T[\mathrm{~K}]$ & MH73 & H74 & M76 & PEVE & EUNIS07 \\
F10.7 cm & & 177 & 123 & 102 & 69 & 67 \\
\hline He II 237 & & 0.44 & 0.57 & - & 0.46 & - \\
He II 243 & & 1.1 & 0.95 & - & 0.89 & - \\
He II 304 & & - & $72(\mathrm{bl}, 2348)$ & - & $63(\mathrm{bl}, 2054)$ & $55(1784)$ \\
He II (dbl) 256.3 & & $? 4.1(? 101)$ & $3.0\left(74^{*}\right)$ & - & $2.0(49)$ & - \\
O VI 184.1 & 5.5 & $0.12\left(3.0^{*}\right)$ & - & - & - & - \\
Fe VIII 185.21 & 5.6 & $0.44(11)$ & - & - & $0.35(9)$ & $0.32\left(8^{*}\right)$ \\
Si VII 275.35 & 5.75 & $0.28(7)$ & $0.25\left(6^{*}\right)$ & - & $0.35(9)$ & - \\
Mg VII (bl) 278.40 & 5.8 & $0.34(8)$ & $0.36\left(9^{*}\right)$ & - & $0.33(8)$ & - \\
Fe IX 171.06 & 5.85 & $4.4(109)$ & $4.2\left(104^{*}\right)$ & $4.4(109)$ & $7.0\left(173^{*}\right)$ & - \\
S VIII (bl) 198.53 & 5.9 & $0.23\left(6^{*}\right)$ & - & - & $0.26(6.4)$ & - \\
Fe X 174.53 & 6.0 & $4.6(114)$ & $4.1(101)$ & $4.2(104)$ & $3.7(91)$ & $4.3\left(107^{*}\right)$ \\
Fe X 177.2 & 6.0 & $2.6(64)$ & $2.2(54)$ & $2.6(64)$ & $2.3(57)$ & $2.2\left(55^{*}\right)$ \\
Fe X 184.52 & 6.0 & $1.0(25)$ & $1.2(30)$ & $1.0(25)$ & $1.2(30)$ & $1.1\left(27^{*}\right)$ \\
Fe X (bl) 190.0 & 6.0 & $0.53(13)$ & $0.55(14)$ & $0.57(14)$ & $0.65(16)$ & $0.51\left(12.5^{*}\right)$ \\
Fe XII 193.50 & 6.1 & $3.2(80)$ & $3.1(77)$ & $2.4(59)$ & $1.0(25)$ & $0.88\left(21.7^{*}\right)$ \\
\hline
\end{tabular}

Notes. The columns indicate the main ion and wavelength $(\AA)$, the approximate formation temperature $(\log T[\mathrm{~K}])$, then the $\mathrm{MH} 73, \mathrm{H} 74, \mathrm{M} 76$, and PEVE irradiances $\left(10^{8}\right.$ phot cm $\left.\mathrm{s}^{-1}\right)$. The values in brackets indicate the estimated QS radiances (phot cm $\mathrm{cm}^{-1} \operatorname{arcsec}^{-2}$ ). The last column gives the EUNIS07 measured QS radiances (in brackets), and the QS irradiances estimated from them. The $10.7 \mathrm{~cm}^{\text {radio }}$ flux in $10^{-22} \mathrm{~W} \mathrm{~m}^{-2} \mathrm{~Hz}^{-1}$ is also shown. The values with an asterisk are those chosen as reference.

again. Then there are the recent EUNIS 2007 rocket flight measurements of Wang et al. (2011), which were instead calibrated on the ground. These values are reported in Table 2. Overall, there is good agreement between the EUNIS 2007 radiances and those obtained from historical irradiances. The values with a star in Table 2 are those adopted here for comparison with the EIS radiances. There is also good agreement among the various irradiances, with the notable exception of the strong Fe IX $171.06 \AA$ and the deblended He II $256.3 \AA$ lines. The variations in the He II are most likely caused by the different solar activity (Del Zanna \& Andretta 2011), but the Fe IX is a puzzle with PEVE providing higher irradiances. Various checks have been made on the EUV lines observed by PEVE, and no problems in the calibration have been found. So the PEVE measurement has been adopted, considering the thorough calibration work that was done before and after that flight.

The EIS QS radiances for a selection of TR lines for which we have previous measurements are shown in Fig. 3. They were obtained with the ground calibration, and clearly show values that are lower (by 20-30 \%) than previous data for most wavelengths, even at the beginning of the mission, when the Sun was very quiet. We recall that the EUNIS 2007 measurements were done during the very low minimum, so these radiances should represent the lowest QS values. The only explanation for the discrepancies is that at the wavelengths displayed the EIS sensitivity in 2007 had already decreased compared to the pre-flight values, as already suggested by Wang et al. (2011). We will return to this issue below.

\subsection{SW and $L W$ line ratios}

Figures 4 and 5 show a selection of line ratios within each SW and LW channel, again using the ground calibration. Bars indicate the predicted values for two different densities, log $\mathrm{Ne}$ $\left[\mathrm{cm}^{-3}\right]=8$ (full lines) and $\log \mathrm{Ne}\left[\mathrm{cm}^{-3}\right]=9$ (dashed lines). The line ratios from AR observations (both on-disk and off-limb cases have been considered) are shown in Fig. 6. More branching ratios and better $\mathrm{S} / \mathrm{N}$ are offered by $\mathrm{AR}$ spectra.
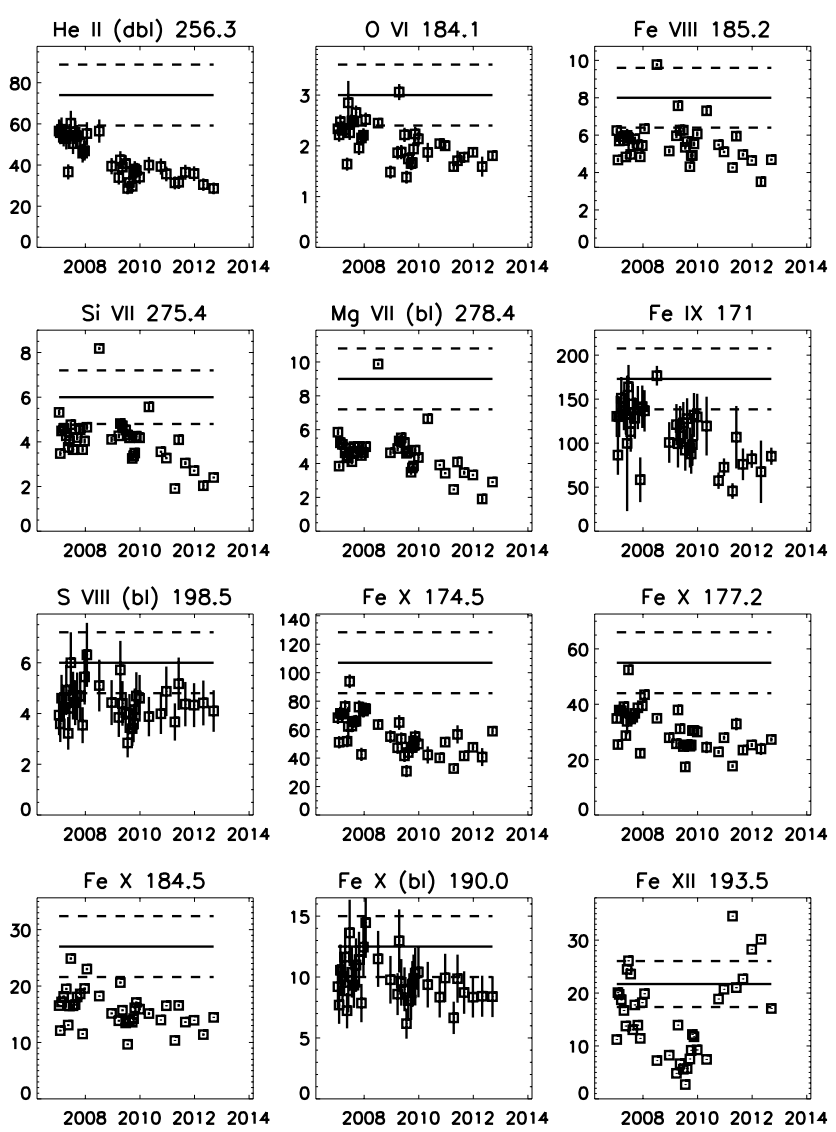

Fig. 3. Averaged EIS QS radiances (phot $\mathrm{cm}^{-2} \mathrm{~s}^{-1} \operatorname{arcsec}^{-2}$ ) obtained with the ground calibration. The lines indicate the QS radiances listed in Table 2 (the dashed lines with a $\pm 20 \%$ ). The He II $256.3 \AA$ line has been deblended.

These line ratios hold a lot of information. Firstly, among those that are expected to be constant in time, most of them do not show significant trends. This is a strong indication that for 

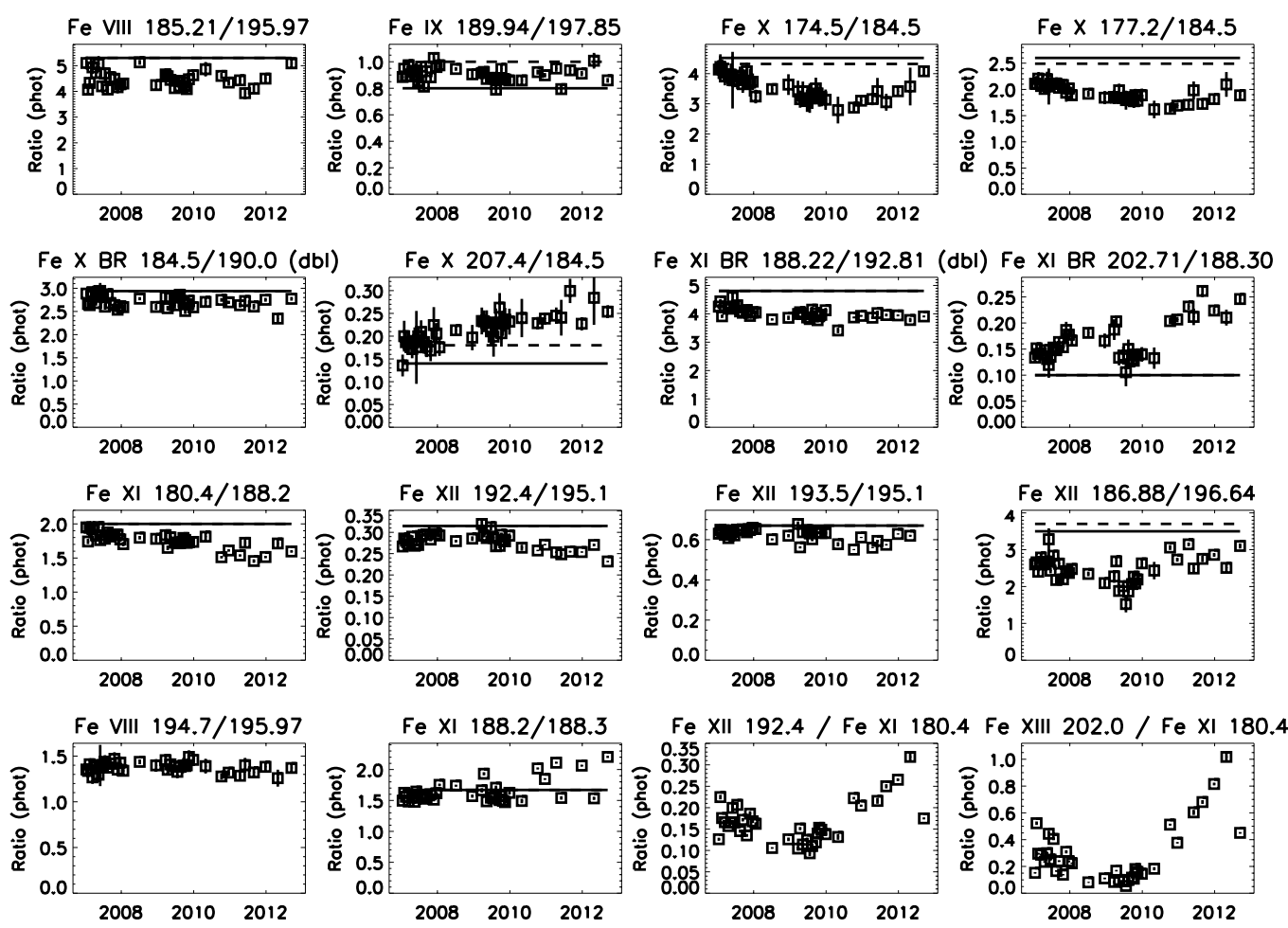

Fig. 4. Ratios of a few SW QS line radiances (photon units) using the ground calibration. Bars indicate the predicted values.

most wavelengths the relative sensitivities within each SW and LW channel have not changed over time. This supports the analysis performed in the following section, where the shapes of the effective areas are obtained for the beginning of the mission from observations during the first two years. Secondly, the small scatter in many of them confirms the correct identification and absence of blending (or correct deblending applied whenever the case). Thirdly, the ratios are generally in good agreement with theory, which gives confidence in both the atomic data and the accuracy of the EIS effective area curves within its stated uncertainty $(22 \%)$ for many wavelengths. The ratios indicate that the shorter wavelengths of both channels had degraded, since they are consistently lower than expected.

Clearly, some ratios show puzzling departures. A few SW ratios such as the Fe X 174.5/184.5 $\AA$, the Fe X 177.2/184.5 $\AA$, and the Fe XI 180.4/188.2 $\AA$ in Fig. 4 show a decrease suggesting that a further degradation occurred in the 170-184 $\AA$ region at least in the first few years. Other ratios we looked at have significant departures beginning in 2010, such as the branching ratio Fe XI 202.71/188.3 $\AA$ shown in Fig. 4. In this case, either the Fe XI 202.71 $\AA$ becomes blended or the $188.3 \AA$ decreases its intensity (or both). The same figure shows that the Fe XI 188.2/188.3 $\AA$ is relatively constant (but with large scatter), so it is possible that the Fe XI 202.71 $\AA$ lines becomes blended with a higher temperature line from Fe XII or Fe XIII for example. Fe XIV lines are excluded because they are comparatively weak in the QS data. Figure 4 shows in the bottom-right corner that Fe XII/Fe XI and Fe XIII/Fe XI ratios increase significantly after 2010 , because the QS solar corona is affected by the solar cycle.

As in the QS case, some AR ratios that are expected to be constant are not. One example is the Fe XIII 200.0/196.5 $\AA$ ratio, shown in Fig. 5. The increase could be due to a line from an ion with higher formation temperature such as Fe XIV. The same figure shows that the Fe XIV $211 \AA$ A/Fe XII $192.4 \AA$ increases after 2010. These two lines are strong and not significantly blended, which means that on average the Fe XIV emission tends to increase compared to the Fe XII in the active region cores.

\subsection{SW/LW ratios and long-term correction}

We now examine the cross-calibration between the SW and LW channels. A selection of ratios in lines in the LW and SW channels is shown in Fig. 7. The line intensities were calculated using the ground calibration. All these ratios, unlike the previous ones, show decreases over time, more marked in the first two years of the mission by a factor of two. The Fe XIV 274.2/211.3 $\AA$, Fe XIII 251.9/201.1 $\AA$, and Fe XI 257.5/188.2 A ratios show a similar trend, within a relative $20 \%$. This is a clear indication of a decrease in the LW/SW relative responsivities, as already seen in Fig. 1. The Fe XIII 201.1 and 211.3 Å lines have been deblended. The Fe XIV ratio is only slightly temperature sensitive, with an expected variation of less than $10 \%$.

The ratios involving lines in the 250-260 A region show observed LW/SW ratios lower than expected (as discussed previously), even at the start of the mission. However, the Fe XIV 274.2/211.3 $\AA$ ratio is close, at the start of the mission, to the expected value. This is another clear indication that the ground calibration should be revised, even for the start of the mission. This is discussed in the next section.

The Fe $\mathrm{X}$ ratio shown in Fig. 7 has some temperature and density dependence; however, the expected variations based on the measured values are small (less than 10\%), and cannot explain the differences with the Fe XI ratio. One explanation could be a decrease in the sensitivity at $184.5 \AA$. This would be in line with the $40 \%$ decrease of the O VI $184.1 \AA$ radiance, but not with the constancy of the $184.5 / 190 \AA$ (and other) ratios.

The O V ratio is not very reliable because the $248.5 \AA$ is very weak and because the ratio varies considerably with density. 

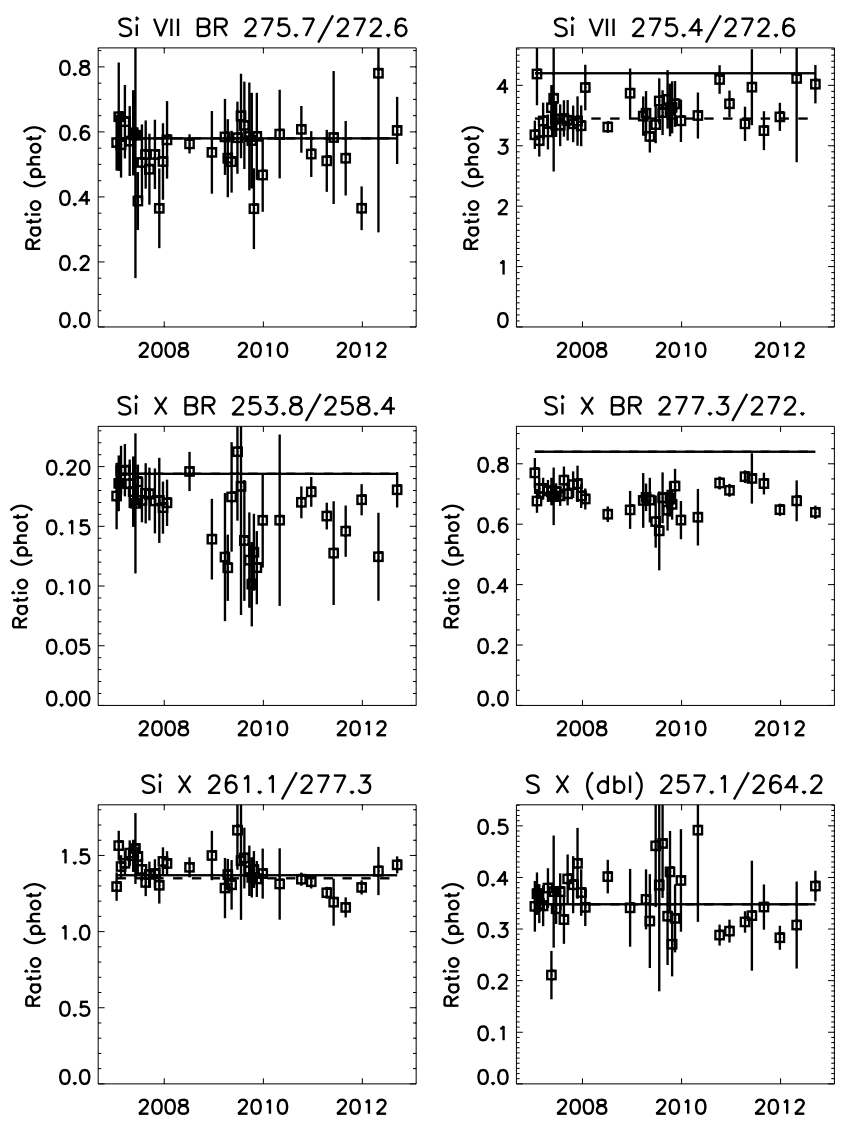

Fig. 5. Ratios of a few LW QS line radiances (in photons) using the ground calibration. Bars indicate the predicted values.

The Si VII $275.3 \AA$ vs. Fe VIII $185.2 \AA$ ratio shows a slightly smaller decrease in time, compared to the other ratios, especially when the Sun becomes active. This ratio was used by Kamio \& Mariska (2012) and Mariska (2013) to argue that both SW and LW channels had the same decrease in sensitivity. Other ratios such as the 278.4/196 $\AA$ show similar behaviour. On the other hand, Mg VI vs. Fe VIII ratios are different. As we have seen, the density and temperature of the QS upper transition region does not seem to change much, so differences in the various ratios should not be expected. The situation is actually quite complex as discussed later.

\subsection{An estimate of the in-flight SW and LW effective areas}

Each observed and theoretical line ratio considered produces a constraint to the relative values of the effective areas. Considering two lines with theoretical intensities $I_{1}$ and $I_{2}$, the ratio of the effective areas is obtained directly from the observed counts: $R_{\text {eff }}\left(\lambda_{1}, \lambda_{2}\right)=E_{1} / E_{2}=I_{2} N_{1} \lambda_{1} /\left(I_{1} N_{2} \lambda_{2}\right)$. Table 1 lists the measured count rates (DN/s) in the pairs of lines, the observed ratio and the derived ratio of the effective areas $R_{\text {eff }}$, compared to that from the ground calibration.

The relative constancy of the various ratios for at least the first two years of the mission means that it is possible to obtain one set of in-flight effective areas that are valid for that period. For each ratio listed in Table 1, we have considered the average over this period. For the ratios involving lower temperature lines we have considered the QS values, while for the hotter temperature lines we considered the AR values, as described previously. The average values are shown in Table 1 . The same table
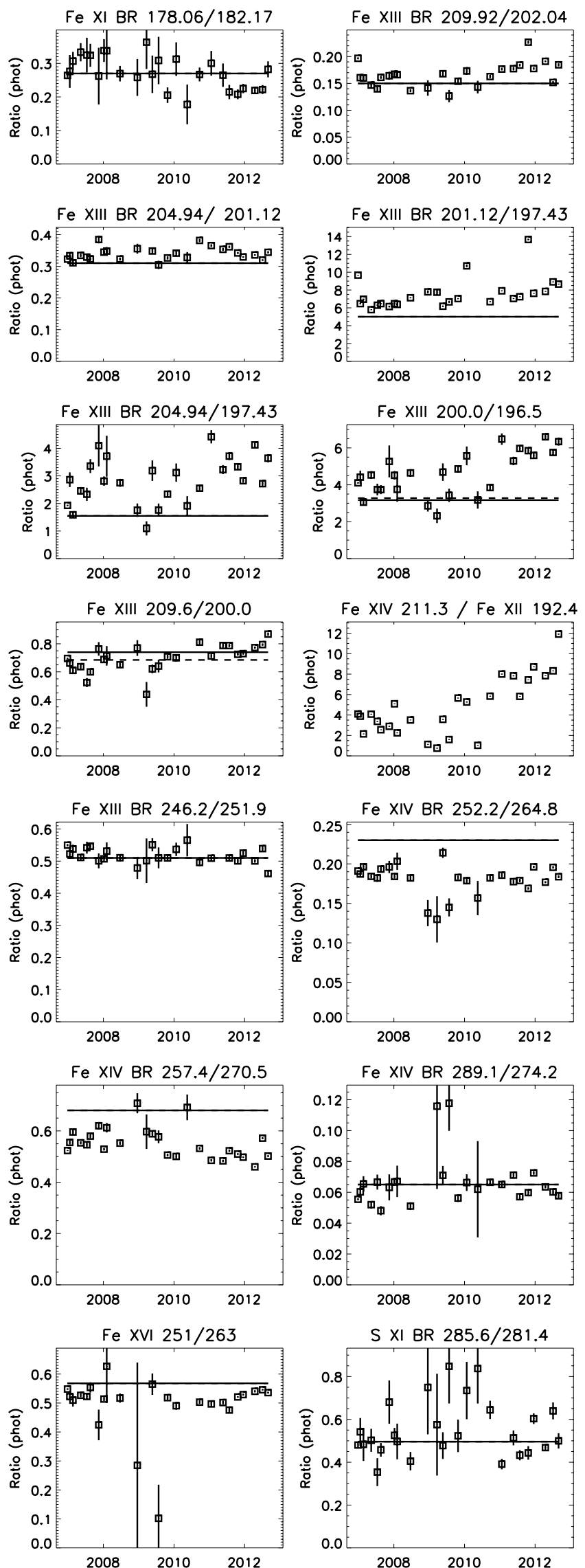

Fig. 6. Ratios of a few AR line radiances (in photons) in the SW and LW channels, obtained with the ground calibration. Bars indicate the predicted values. 

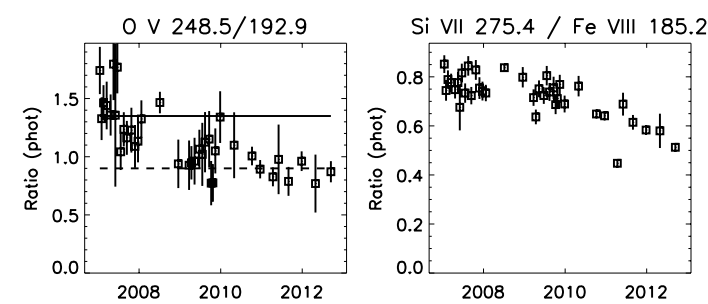

Mg VII+Si VII 278.4 / Fe VIII 196 Mg VI 270.4 / Fe VIII 196
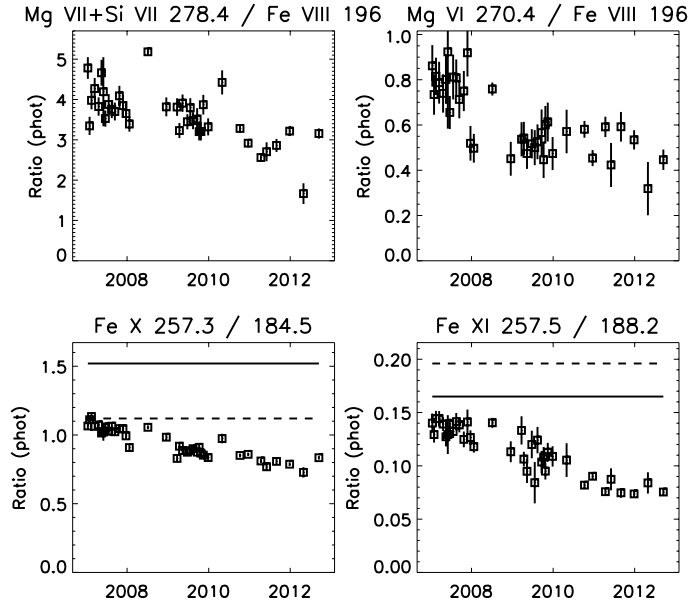

Fe XIII 251.95/201.12

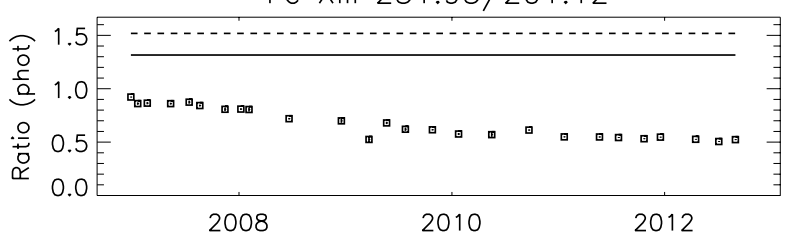

Fe XIV 274.2/211.3

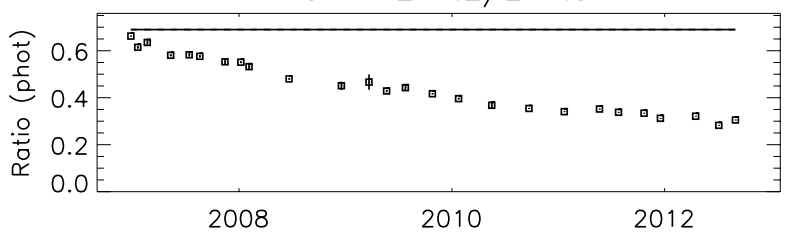

Fig. 7. Ratios of a few LW vs. SW line radiances (in photons) using the ground calibration. Bars indicate the predicted values. The top six plots show the ratios from the QS observations, the lower ones are from the AR observations.

also shows the average count rates in each line forming the ratio. Considering that the exposure time was $90 \mathrm{~s}$, the numbers in the table show that the total counts in most selected lines were high, a few hundred. Hence, single measurements of most lines have a small uncertainty. We have adopted as uncertainty in the ratios one standard deviation from the average. These uncertainties are also listed in Table 1. Finally, for the uncertainties in the ratios of the effective areas $R_{\text {eff }}$ we have summed in quadrature the uncertainties in the averaged observed ratios, and the estimated theoretical uncertainties of the ratios, which are also listed in Table 1.

We have performed the procedure independently for the SW and LW channels. We started with two splines at a selection of nodes and with values such that the curves were identical to the ground calibration curves. We then adjusted the spline node values to satisfy the observed ratios of the effective areas. The final effective areas are shown in Fig. 8 in the top two plots. Each line ratio used is linked with dashed lines, and the combined uncertainties for each ratio overplotted. The values of the spline nodes are listed in the Appendix. The SW effective areas were scaled by assuming that the values near $195 \AA$ (the peak) are the same as those of the ground calibration. The LW effective areas were scaled as described in the following section. The five ground calibration measurements (one for the SW and four for the LW) are also shown, with their $20 \%$ uncertainty.

The results for the SW channel are shown in logarithmic scale. Overall, good agreement with the ground calibration (dashed line) is found, although significant departures in the shorter wavelengths of both channels is present. This result is puzzling, but there is a significant consistency in the various ratios, and in all the subsequent analysis that has been done on several datasets. We note that the discrepancies are not within the estimated uncertainties. We will return to this issue in the conclusions.

\subsection{An estimate of the relative $L W / S W$ effective areas}

The LW/SW relative values in Fig. 8 (bottom plot) were obtained from a combination of measurements. We have used the off-limb QS of 2006 Dec 23 for the lower-temperature lines, the on-disk AR spectra of 2006 Dec 25 for the Fe XIII and Fe XIV line ratios, and the 2007 Jun 2 flare for the Fe XVII and Fe XXIV line ratios. The measurements of the flare lines (listed in Del Zanna 2008) are uncertain because of the offset of about $2^{\prime \prime}$ in the pointing of the SW and LW channels mentioned previously and the dynamic nature of flares. However, the flare was very small and the variations to be expected from the non-simultaneity of the observations cannot explain the discrepancies, which are also found in every later observation after 2008 Aug. 24, as mentioned in the introduction.

In combining the 2006 Dec. and 2007 June datasets we neglect the variation in the relative LW/SW calibration, which is small, however, as described below. The LW effective areas are considerably lower than measured on the ground. As we have seen, they continued to decrease over time.

A detailed emission measure analysis was performed on the off-limb quiet Sun observation of 2006 Dec. 23. Excellent agreement among all the SW and LW lines was found, further confirming the new calibration. The results of this analysis are not presented here since they would require a lengthy discussion of all the blends and the lines. A similar line ratio study was performed with the 2007 Aug. 19 AR observations discussed in detail in Del Zanna (2012). The new effective areas remove all the main discrepancies.

\subsection{The long-term correction}

We recall that the ratios shown in Fig. 7 show similar trends, i.e. a marked decrease in the LW/SW ratios during the first two years of the mission when the Sun was very quiet, and so no significant changes would be expected. To provide an estimate for the long-term drop in sensitivity, we have considered the Fe XIV, Fe XIII, and Fe XI ratios. Each of them has its pros and cons. Active region spectra offer better signal, but a large number of unidentified lines appear there (see the spectral atlas in Del Zanna 2012), so it is possible that additional blending occurs in the lines. Additionally, AR spectra are normally very inhomogeneous, with large variations in temperatures and densities which could affect some of the ratios, even if only slightly. The Fe XIV 211.3/274.2 $\AA$ ratio has the problem that the $211.3 \AA$ line is just at the edge of the SW channel, and the $274.2 \AA$ line 

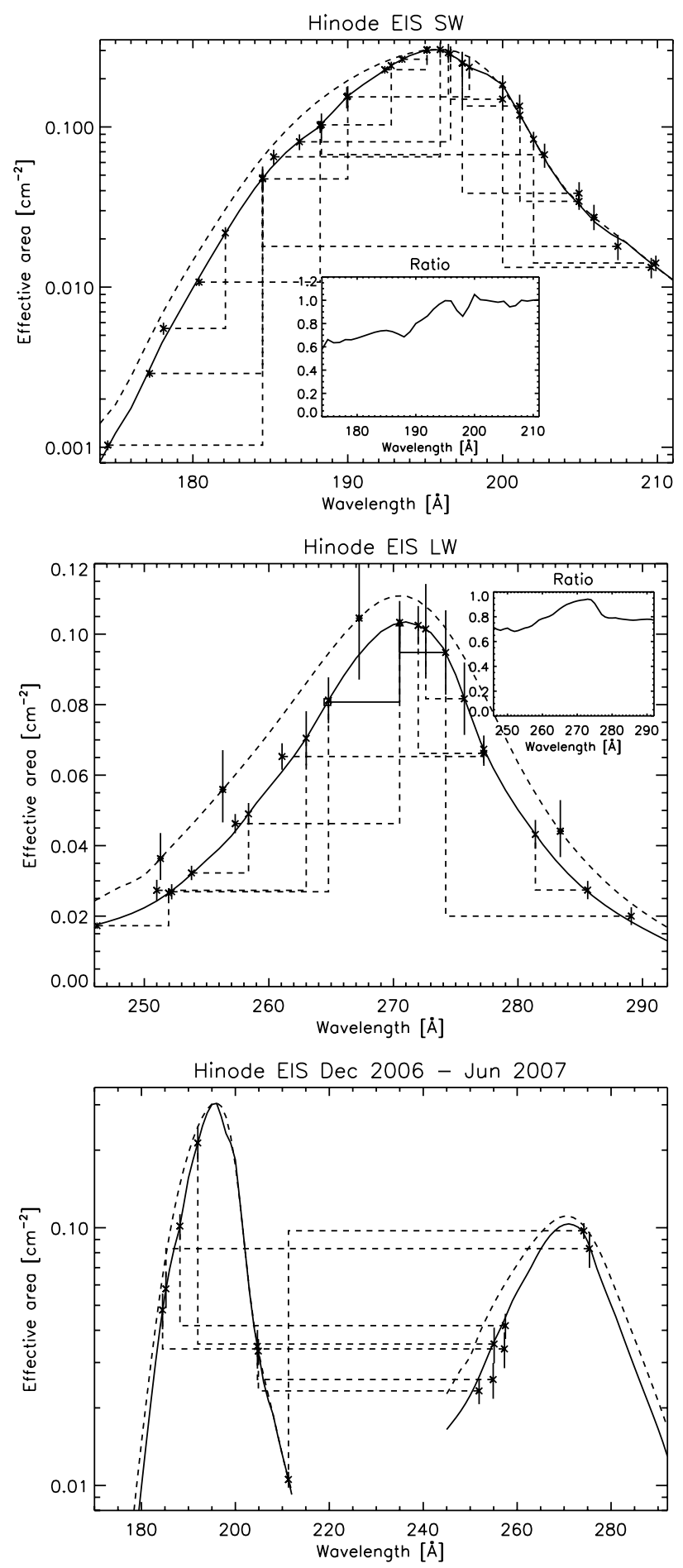

Fig. 8. Effective areas for the EIS channels obtained from the first two years of QS and AR observations analysed here, and the 2007 Jun. 2 flare observation (Del Zanna 2008). The full lines indicate the proposed values, the dashed lines those from the ground calibration. The top two plots show the line ratios used for the SW and LW channels separately, while the bottom plot shows the cross-channel line ratios. The asterisks show the five measurements of the ground calibration. The insets show the ratios between the measured values and those of the ground calibration.

is blended with a Si VII line, which can contribute up to $20 \%$ or so. The Si VII line is density sensitive and has additional uncertainty in its estimation. The QS spectra have less signal, and show larger scatter.

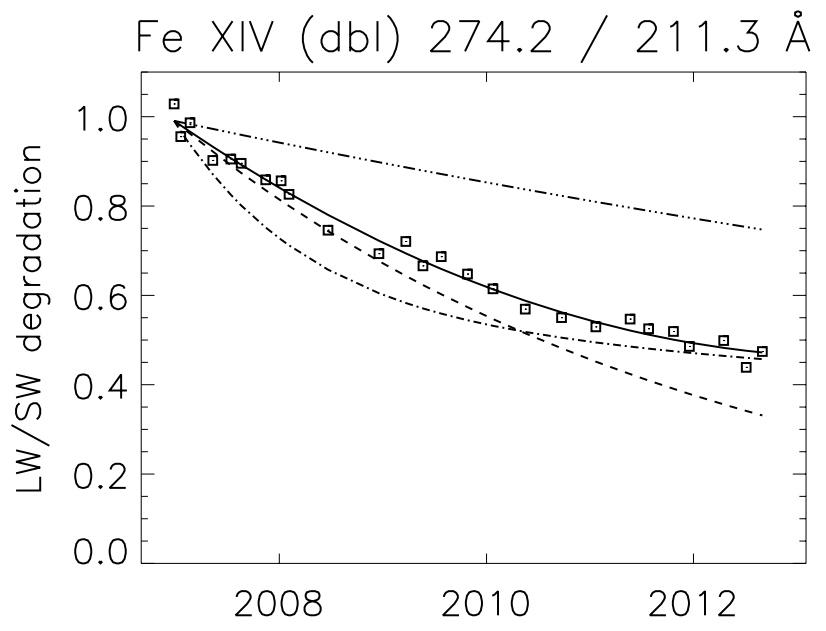

Fig. 9. Observed Fe XIV 274.2/211.3 $\AA$ ratio, divided by its theoretical value. The observed ratio is computed with the present effective areas for the SW and LW channels (Fig. 8). This normalised ratio is used to infer the long-term correction for the decrease in sensitivity of the LW channel. The solid line is the fit, while the other lines are previous suggestions (see text).

To provide an estimate for the long-term drop in sensitivity, we show the results from the Fe XIV 274.2/211.3 $\AA$ ratio in Fig. 9. Similar results are obtained from the Fe XI and Fe XIII ratios. We note that there is agreement only if the recent atomic data for Fe XI are used, and careful deblending of the Fe XIII and Fe XIV is done.

The trend in the Fe XIV 274.2/211.3 $\AA$ ratio is easily measured, but the absolute value of the correction depends on the radiometric calibration and the predicted value for the ratio. We have adopted the present radiometric calibration described in the previous section, and recall that we assumed a predicted value of 0.69 for this ratio. We note that the ground calibration provides similar values. The ratio points have been fitted with a polynomial curve, also shown in Fig. 9. This curve is assumed as our long-term correction for the degradation of the LW channel (details are given in the Appendix).

The three other curves in Fig. 9 show for comparison the exponential decays as available within the EIS software (dashed line), as suggested by Kamio \& Mariska (2012) (dot-dashed line), and by Mariska (2013) (triple dot-dashed line). These exponential decays have been normalised to the first point. We recall that both Kamio \& Mariska (2012) and Mariska (2013) assumed the same decay rate for both SW and LW. For the purposes of this plot these results have been interpreted as only applying to the LW channel. We note that there are clear differences in the behaviour of the four corrections.

As a first-order calibration we assume in the remainder that: a) the shapes of the SW and LW effective areas are as shown in Fig. 8 and do not change over time; b) the SW sensitivity does not change over time; and c) the LW effective areas decrease over time as is shown by the fit in Fig. 9. The parameters for the polynomial fit are given in Appendix B.

\subsection{The first-order calibration applied to a few cases}

We have applied the present first-order calibration to several cases spread over the years. We have found good overall agreement between observations and theory. Some examples are given below. We start by showing in Fig. 10 the QS count rates in a few LW lines, corrected for the LW long-term decrease in sensitivity. 

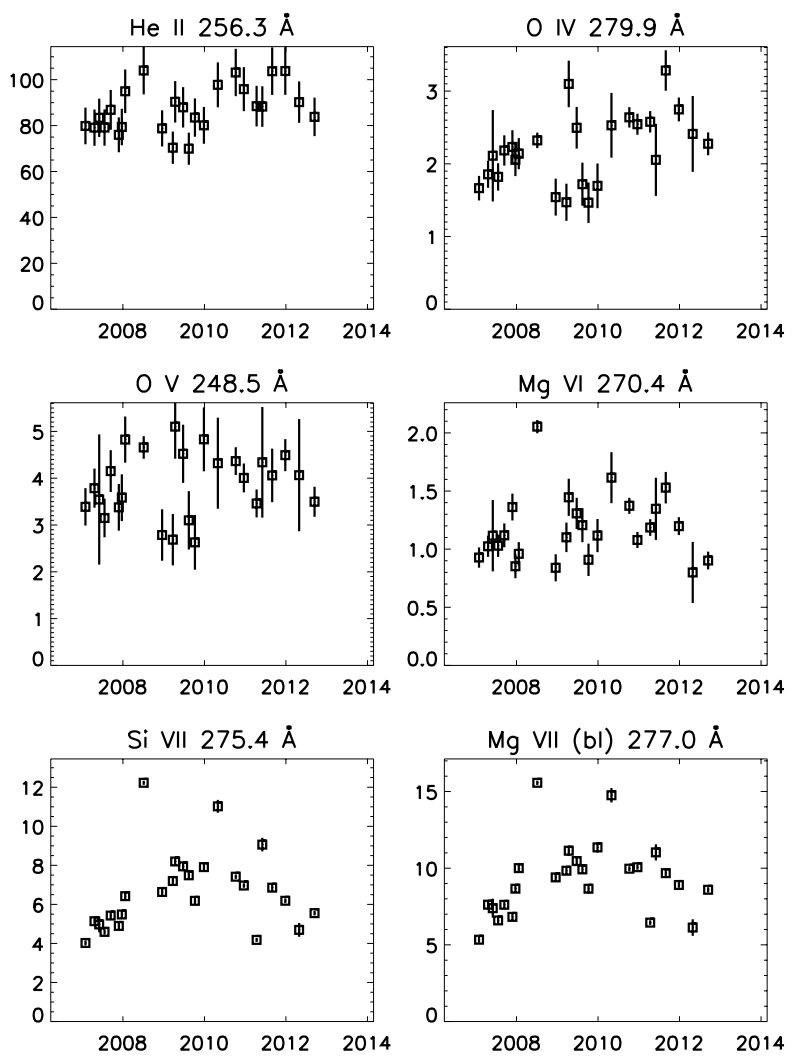

Fig. 10. Averaged EIS count rates (DN/s per 1") in the QS areas, corrected for the LW long-term decrease in sensitivity.

The figure shows that we obtain relatively constant values in the cool lines, most notably the He II $256.3 \AA$ line (which has been deblended), but also the O IV, O V, and Mg VI lines. This confirms the reliability of the long-term correction. Some residual variations and a large scatter are present, but they are due to solar variability. We recall that the synoptic observations were carried out on small fields of view and are therefore not ideal for carrying out a proper study of the solar radiances.

Figure 11 shows the EIS QS calibrated radiances of the same lines shown in Fig. 3 (those for which we found QS historical values), obtained with the present first-order calibration, i.e. both new effective areas and long-term correction. There is clearly a very good agreement (to within a relative $\pm 20 \%$ ) with the previous measurements during the first two years of the mission when the Sun was quiet.

Figure 12 shows the ratios of a few QS and AR line radiances (in photons) in the SW and LW channels, obtained with the present first-order calibration. The $\mathrm{O} v$ ratio shows an overall constancy. The correction based on the Fe XIV ratio reproduces the Fe XIII ratio extremely well and the Fe XI 257.5/188.2 A ratio reasonably well. The increase in Fe X 257.3/184.5 $\AA$ is puzzling. If density variations are neglected, the trend would imply a decrease in the temperature, which is contrary to what is observed. The increase could partly be ascribed to a decrease in the effective area at $184.5 \AA$ not taken into account. The Si VII $275.4 \AA / F e$ VIII $185.2 \AA$ corrected ratio shows a residual increase over time. We discuss this in the following section.

\subsection{The question of the Si VII vs. Fe VIII}

We recall that the Si VII $275.3 \AA$ vs. Fe VIII $185.2 \AA$ ratio was used by Kamio \& Mariska (2012) and Mariska (2013) to argue
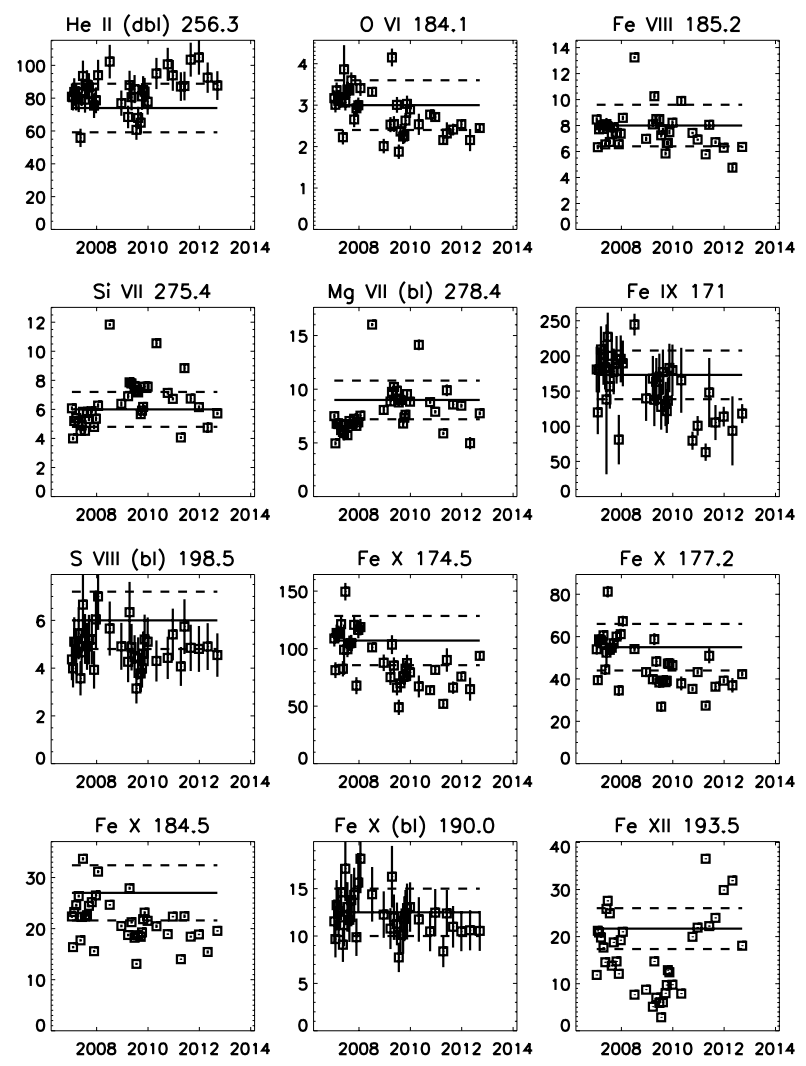

Fig. 11. Averaged EIS QS radiances as in Fig. 3, but obtained with the present calibration.

that both SW and LW channels had a similar degradation. There are two problems with using this ratio, however. First, the Si VII and Fe VIII ions are probably not formed in the same spatial regions. This is because the temperature of formation of Si VII is different from that of Fe VIII (although the predicted Fe VIII formation temperature has changed significantly over the years because of improvements in the calculations and measurements of the ionisation and recombination rates). The second problem is that some variations in the iron vs. silicon abundances, although not expected on a large scale, could be happening. Both effects might be the reason for the large scatter in the ratios of the Si VII and Fe VIII lines shown in Fig. 7. We note that the scatter is not limited to the two lines, because all the Fe VIII lines and all the Si VII lines have similar trends, i.e. their ratios are relatively constant, as shown in the previous figures.

It is also clear that other ratios involving the $\mathrm{Mg}$ VI and Fe VIII lines behave in a different way, as shown in Fig. 12 with the Mg VI 270.4/Fe VIII $196 \AA$ ratio. There is still scatter but the ratios are fairly constant once the present long-term correction is applied. We have checked that all the Mg VI lines in the LW channel behave in the same way. The puzzling issue is that the Mg VI lines are close in wavelength to the Si VII lines. The fact that the Mg VI vs. Fe VIII ratios show less scatter and a different trend over time, compared to the Si VII vs. Fe VIII ratios, might be because the TR components of Mg VI and Fe VIII originate from the same temperatures/spatial regions. This is discussed in the Appendix in more detail.

\subsection{Observations in 2012}

Several recent observations in 2012 were then analysed to see if this first-order calibration is sufficient to remove the main 

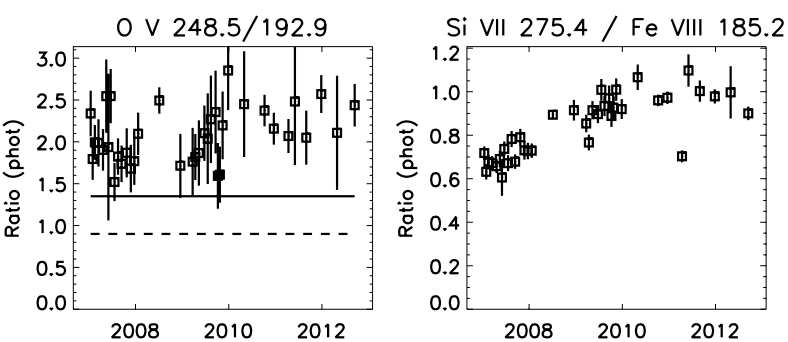

Mg VII+Si VII 278.4 / Mg VI 270.4 Mg VI 270.4 / Fe VIII 196
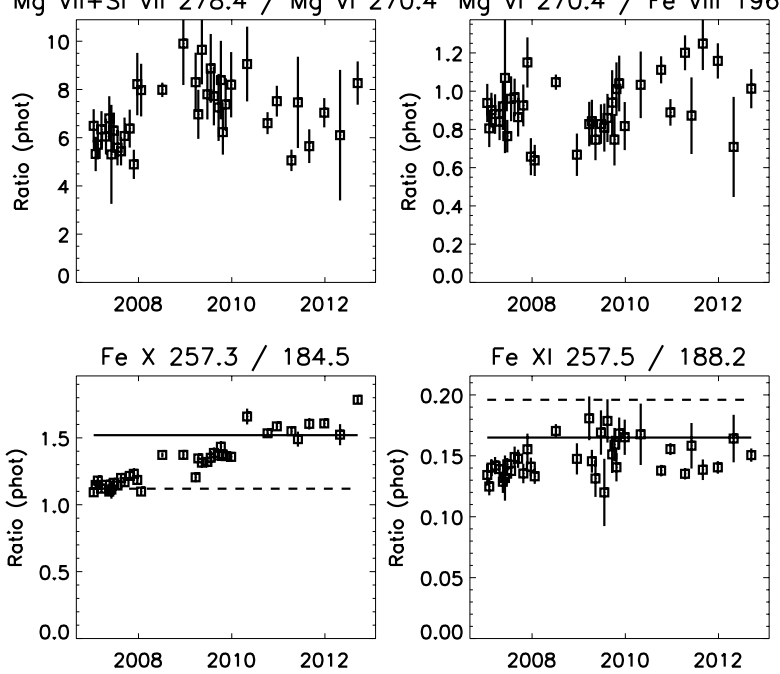

Fe XI $257.5 / 188.2$
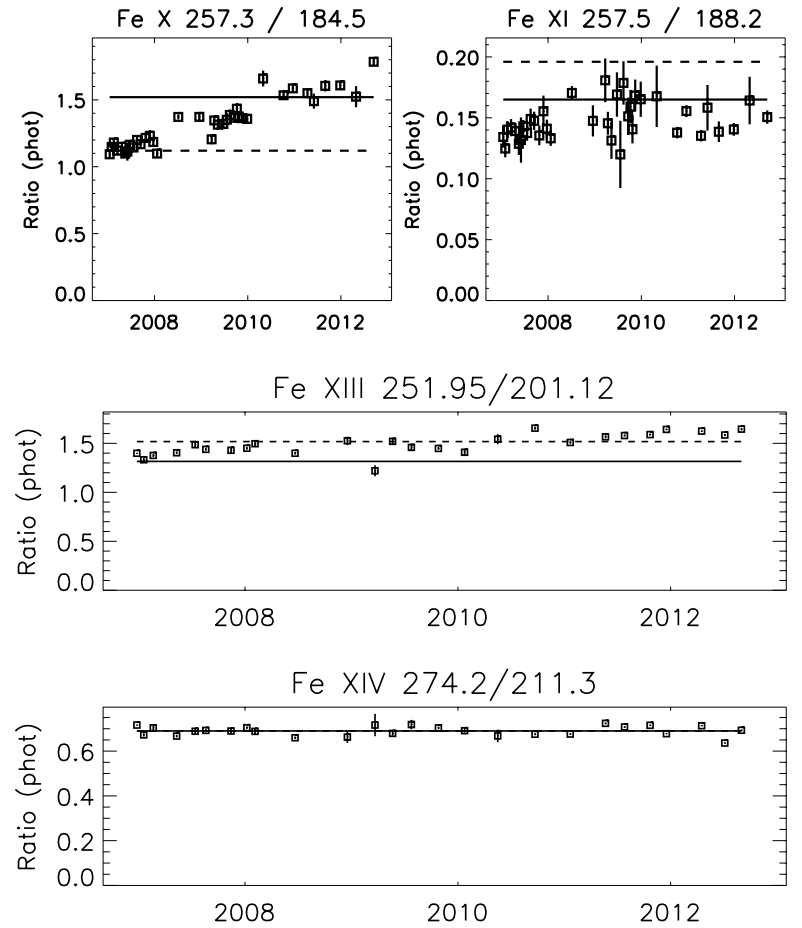

Fig. 12. Ratios of a few $Q S$ and $A R$ line radiances (in photons) in the SW and LW channels, obtained with the present first-order calibration. Bars indicate the predicted values.

problems. We present here only the results of the analysis of an off-limb AR observation of 2012 Apr. 16 and a flare observation of 2012 Mar. 9. A preliminary analysis of the 2012 Apr. 16 observation indicates that large departures in the shapes of the effective areas have not occurred, with the exception of the shorter wavelengths of the SW channel, where the sensitivity has further decreased. This is all consistent with the behaviour of the line ratios discussed previously.

The 2012 Mar 9 observation is particularly important since it is the first and only EIS full-spectral observation of a mediumsize M-class flare. A detailed description of this observation is presented in a separate paper. Here, we focus on the Fe XVII and Fe XXIV lines. Extra care was needed to select the best spectra for either Fe XVII or Fe XXIV. For example, various lines were saturated in many exposures. The Fe XXIV lines during

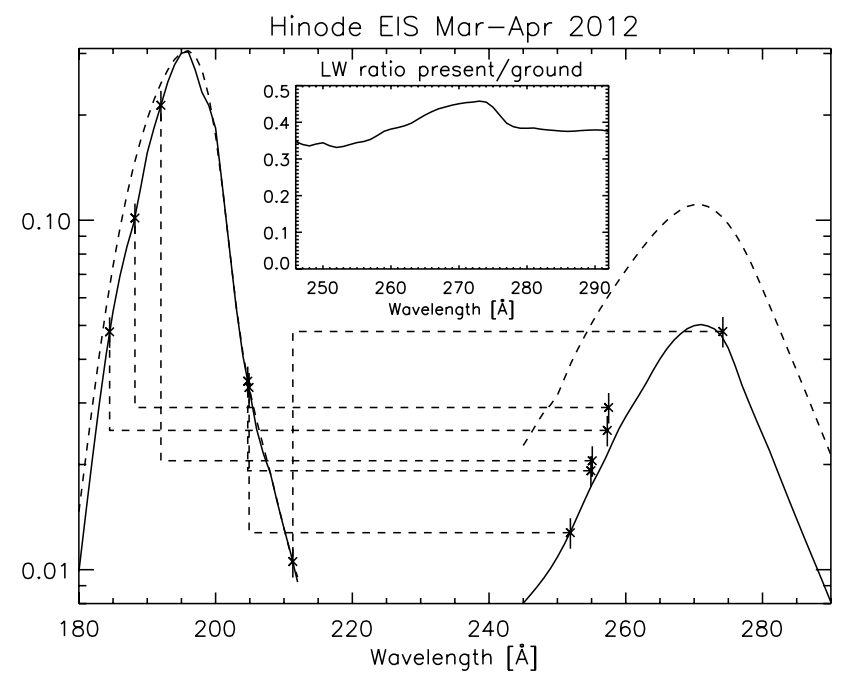

Fig. 13. Effective areas for the EIS channels obtained with the first-order calibration for the period 2012 Mar.-Apr. The full lines indicate the proposed values, the dashed lines those from the ground calibration. The ratios of the effective areas as obtained from the off-limb AR observation of 2012 Apr. 16 and the flare observation of 2012 Mar. 9 are overplotted, showing good agreement.

the impulsive phase have large blue-shifted components which complicate the analysis. One spectrum for Fe XVII and one for Fe XXIV was obtained. In the first spectrum, the Fe XVII lines are so bright that significant blending should not be present. The Fe XVII lines are very useful for checking the calibration because they fall across most LW wavelengths. Discrepancies of over a factor of two were found in the Fe XVII 204.7/254.9 $\AA$ and Fe XXIV 192/255.1 $\AA$ ratios using the ground calibration. The first-order calibration presented above removes these main discrepancies.

The 2012 March and 2012 April line ratios have been combined in Fig. 13 by applying the first-order correction. The longterm correction indicates a decrease of about a factor of two in the relative LW/SW sensitivity for this period. The very reliable Fe XVII and Fe XXIV ratios show very good agreement with the present correction (see points at 254.9 and $255 \AA$ ). In the Fe XXIV spectrum, the Fe XXIV 192/255.1 $\AA$ ratio is about 4 (photon units) if the ground calibration is used, instead of the expected 1.85. The present first-order calibration brings the ratio down to a value of 1.6, i.e. within a reasonable $15 \%$ of the expected value. The agreement in the ratios involving lines shortward of $190 \AA$ can be improved if a wavelength-dependent correction for the SW channel is introduced.

Figure 14 shows the emissivity ratio curves relative to the main Fe XVII lines. These emissivity ratio curves are obtained by dividing the observed intensity $I_{\mathrm{ob}}$ of a line by its emissivity

$F_{j i}=\frac{I_{\mathrm{ob}} N_{\mathrm{e}} C}{N_{j}\left(N_{\mathrm{e}}, T_{\mathrm{e}}\right) A_{j i}}$,

calculated at a fixed electron density $N_{\mathrm{e}}$ and plotted as a function of the temperature $T_{\mathrm{e}}$ (see Del Zanna et al. 2004 for details). The scaling constant $C$ is chosen so that the $254.9 \AA$ curve is close to unity.

The emissivity ratio curves show the large discrepancy (more than a factor of two) in the Fe XVII 204.7/254.9 $\AA$ branching ratio, when the current ground calibration is adopted (top plot). The present first-order calibration brings the ratio within an excellent $10 \%$. With the new calibration, there is very good 

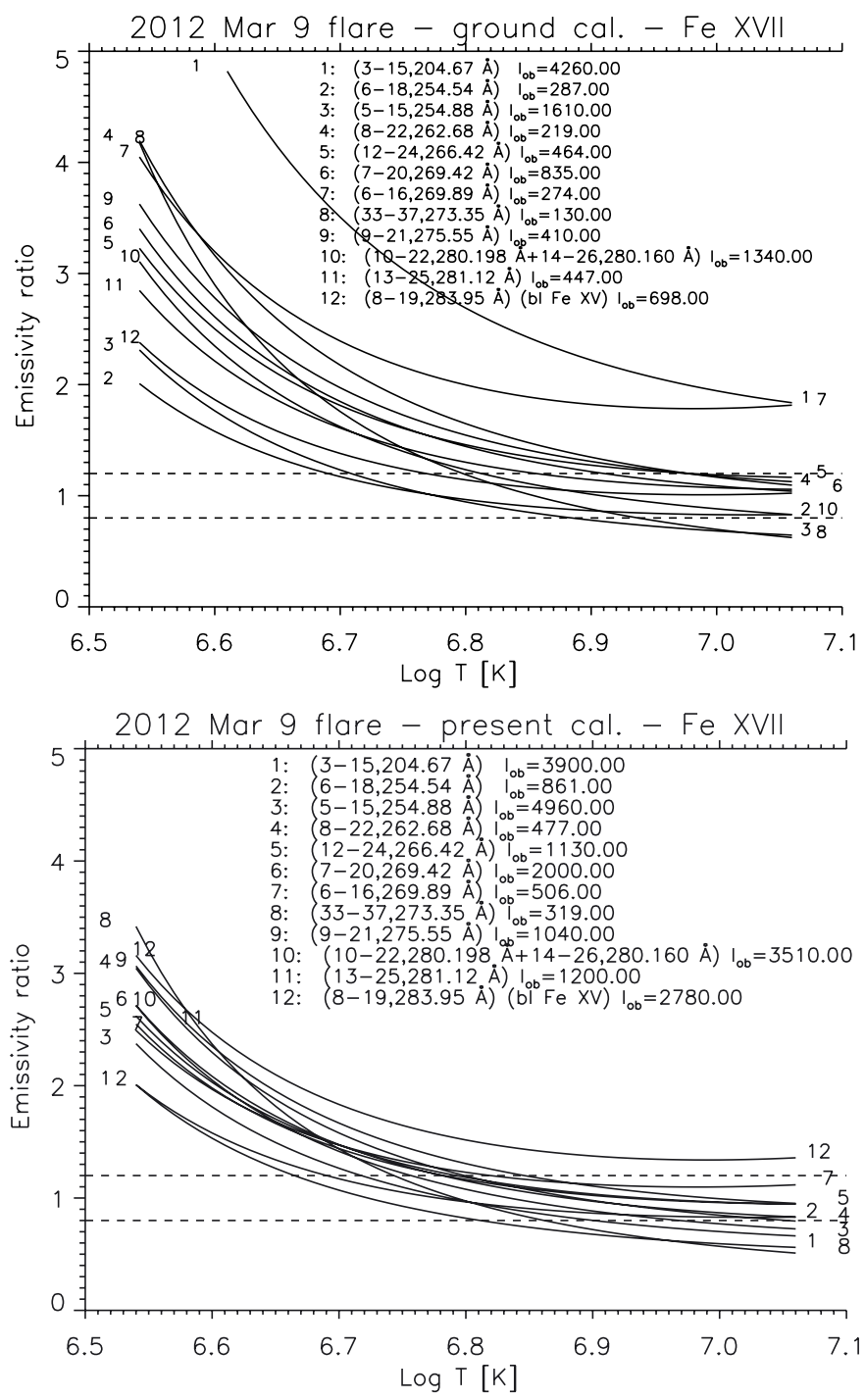

Fig. 14. Emissivity ratio curves relative to the main Fe XVII EUV transitions observed by Hinode EIS on 2012 Mar. 9. $I_{\mathrm{ob}}$ is the calibrated observed intensity in photons $\mathrm{cm}^{-2} \mathrm{~s}^{-1} \operatorname{arcsec}^{-2}$. The top plot shows the results with the current ground calibration, the bottom plot with the present first-order correction.

agreement (to within a relative $20 \%$ ) between theory and observation for all the Fe XVII lines, a remarkable result which further confirms the present calibration. The only line with a significant departure is very close to the Fe XV $284.1 \AA$ line and is difficult to measure it accurately. On the other hand, large discrepancies are present if the ground calibration is adopted. All the Fe XVII lines are very strong, but some small blending is expected to be present. Further refinements will need an indepth analysis that is left to a separate paper. The plot also shows that the ratio of the Fe XVII $273.35 \AA$ line with any of the other lines can potentially be used to measure electron temperatures for flares.

\section{Conclusions}

This preliminary assessment of the in-flight degradation of the EIS instrument based on line radiances and ratios shows a consistent pattern that is fundamentally different from what has been assumed so far.

The observations show a clear degradation of the longwavelength channel, compared to the short-wavelength channel.
The responsivity of the $\mathrm{LW}$ channel, at its shorter wavelengths, in 2006 December was already lower (by 20-30\%, see inset in the middle plot in Fig. 8) than the same responsivity measured on the ground. It continued to decrease significantly until 2010 when radiances in LW lines became underestimated by a factor of 2. On the other hand, QS radiances in SW lines do not show any indication of a major degradation for the SW channel, with the exception of its shorter wavelengths.

Overall, the shapes of the effective areas in their central regions are close to those measured on the ground. Significant departures (30-50\%) are present towards the edges of both SW and LW channels, however. Such departures are not surprising, especially considering the large discrepancies (up to 50\%) within the ground measurements, discussed previously. The present LW long-term correction brings the main LW/SW line ratios to become constant to within a reasonable $20 \%$, and produces relatively constant QS radiances in the LW cool lines, most notably the strongest line, He II $256.3 \AA$.

The present new calibration also removes a number of peculiar features in terms of emission measures and elemental abundances that we have encountered. A significant number of studies such as those concerning emission measures combine the use of EIS cooler lines (e.g. Fe VIII-Fe XIII) from the SW channel with the hotter ones (Fe XIV-Fe XVI) from the LW channel. These studies should be revised by taking into account the decrease in the responsivity of the LW channel.

The EIS instrument has performed reasonably well, with a clear degradation of only a factor of two in one of the channels within the first two years of the mission. We note that significant degradations (one order of magnitude) are very common, even in recently-built instruments, especially if they have a front filter. For example, already at first light, the SDO EVE MEGS-B showed a factor of 10 drop in sensitivity, and significant decreases are still occurring at selected wavelengths. Such degradations are measured by using different filters, some only occasionally for calibration purposes. Different front filters show very different attenuations. The SDO EVE ESP instrument has an Al filter, and shows similar attenuation as the MEGS-A2 instrument, which has an Al-Ge-C filter. The MEGS-A2 shows a clear wavelength dependence, with more attenuated lines at longer wavelengths. After two years, lines around $190 \AA$ were attenuated by $30 \%$, while those at $284 \AA$ by $50 \%$. This is interpreted as carbon deposition in the front filter. The LYRA instruments on-board PROBA-2 also suffered large degradations, known to be caused by contamination (probably molecular) on the front optical filters.

Other instruments such as the SOHO CDS performed much better, with a degradation of only a factor of 2 over 13 years (Del Zanna et al. 2010a), perhaps because of the rigorous cleanliness program and/or because of the lack of an entrance filter. Such calibration issues were discussed at the on-orbit degradation workshop that took place in the Solar Terrestrial Centre of Excellence (STCE, Royal Observatory of Belgium) in Brussels on May 3, 2012. A summary is given in BenMoussa et al. (2013). As a follow-up, further meetings will be hosted by STCE to check the inter-calibrations of the various instruments.

Out-gassing and contamination of the entrance filter could be partly responsible for the observed degradation in the EIS LW channel. Deposition of carbon compounds would cause an enhanced degradation in the LW channel, compared to the SW channel, as observed in the SDO EVE MEGS-A2. However, they would also cause some degradation in the SW channel. We have seen some evidence of degradation at the SW shorter 
wavelengths but not at the longer ones, which is not expected. Another possible cause of the lower sensitivity of the LW channel could be a degradation of the $\mathrm{Mo} / \mathrm{Si}$ multilayer (Jones, priv. comm.). The present calibration is a contribution to the ongoing effort within the EIS team to understand the degradation of the instrument and to provide the best possible correction.

A preliminary comparison between EIS and EVE data since 2010 May has shown very similar results to those presented here (Ugarte Urra \& Warren, priv. comm.). All the results shown in Mariska (2013) are also in agreement with those shown here, although the interpretation is very different. Mariska (2013) assumes that there is something odd about the He II $256.3 \AA$ line, and that the LW/SW calibration can be checked using the Si VII $275.4 \AA$ vs. Fe VIII $185 \AA$ ratio. We have seen the various problems associated with this ratio. The suggestion from Mariska (2013) does not explain the factor of two problems with the Fe XI, Fe XIII, Fe XIV, Fe XVII, and Fe XXIV lines observed in the SW and LW channels in the data after 2010. The present calibration resolves all the discrepancies, but leaves somewhat unexplained the behaviour of some lines. Further work can be done on the lines of the present study; however, what is needed is a calibration rocket. In the near future, a new EUNIS rocket should be launched, which will provide important information on the current EIS calibration.

Acknowledgements. Useful discussions and comments from various members of the EIS team are acknowledged, in particular from J. Mariska, T. Watanabe, H. Hara, and H. Warren. Special thanks go to the referee, P. Young, for detailed discussions and suggestions on how to improve the manuscript. Support from STFC is acknowledged. The work of the UK APAP Network was funded by the UK STFC under grant No. PP/E001254/1 with the University of Strathclyde. CHIANTI is a collaborative project involving researchers at the University of Cambridge (UK), the George Mason University, and the University of Michigan (USA). The excellent Hinode Science Data Centre Europe was used to search the EIS database. Hinode is a Japanese mission developed and launched by ISAS/JAXA, with NAOJ as domestic partner and NASA and STFC (UK) as international partners. It is operated by these agencies in co-operation with ESA and NSC (Norway).

\section{References}

Andretta, V., \& Del Zanna, G. 2013, A\&A, submitted

Andretta, V., Del Zanna, G., \& Jordan, S. D. 2003, A\&A, 400, 737

BenMoussa, A., Gissot, S., Schühle, U., et al. 2013, Sol. Phys., in press [arXiv: 1304 . 5488]

Brosius, J. W., Davila, J. M., \& Thomas, R. J. 1998, ApJ, 497, L113

Chamberlin, P. C., Woods, T. N., Crotser, D. A., et al. 2009, Geophys. Res. Lett., 36, 5102

Culhane, J. L., Harra, L. K., James, A. M., et al. 2007, Sol. Phys., 60

Del Zanna, G. 1999, Ph.D. Thesis, Univ. of Central Lancashire, UK
Del Zanna, G. 2003, A\&A, 406, L5

Del Zanna, G. 2008, A\&A, 481, L69

Del Zanna, G. 2009a, A\&A, 508, 501

Del Zanna, G. 2009b, A\&A, 508, 513

Del Zanna, G. 2010, A\&A, 514, A41

Del Zanna, G. 2011, A\&A, 533, A12

Del Zanna, G. 2012, A\&A, 537, A38

Del Zanna, G., \& Andretta, V. 2011, A\&A, 528, A139

Del Zanna, G., \& Ishikawa, Y. 2009, A\&A, 508, 1517

Del Zanna, G., \& Mason, H. E. 2005, A\&A, 433, 731

Del Zanna, G., \& Storey, P. J. 2012, A\&A, 543, A144

Del Zanna, G., \& Storey, P. J. 2013, A\&A, 549, A42

Del Zanna, G., Bromage, B. J. I., Landi, E., \& Landini, M. 2001, A\&A, 379, 708

Del Zanna, G., Berrington, K. A., \& Mason, H. E. 2004, A\&A, 422, 731

Del Zanna, G., Andretta, V., Chamberlin, P. C., Woods, T. N., \& Thompson, W. T. 2010a, A\&A, 518, A49

Del Zanna, G., Storey, P. J., \& Mason, H. E. 2010b, A\&A, 514, A40

Del Zanna, G., Mitra-Kraev, U., Bradshaw, S. J., Mason, H. E., \& Asai, A. 2011, A\&A, 526, A1

Del Zanna, G., Storey, P. J., Badnell, N. R., \& Mason, H. E. 2012a, A\&A, 543, A139

Del Zanna, G., Storey, P. J., Badnell, N. R., \& Mason, H. E. 2012b, A\&A, 541, A90

Dere, K. P., Landi, E., Mason, H. E., Monsignori Fossi, B. C., \& Young, P. R. 1997, A\&AS, 125, 149

Hara, H., Watanabe, T., Harra, L. K., Culhane, J. L., \& Young, P. R. 2011, ApJ, 741,107

Haugan, S. V. H. 1997, CDS software note, 47

Heroux, L., Cohen, M., \& Higgins, J. E. 1974, J. Geophys. Res., 79, 5237

Kamio, S., \& Mariska, J. T. 2012, Sol. Phys., 279, 419

Landi, E., Del Zanna, G., Young, P. R., Dere, K. P., \& Mason, H. E. 2012, ApJ, 744,99

Lang, J., Kent, B. J., Paustian, W., et al. 2006, Appl. Opt., 45, 8689

Liang, G. Y., \& Badnell, N. R. 2010, A\&A, 518, A64

Liang, G. Y., Whiteford, A. D., \& Badnell, N. R. 2009a, A\&A, 499, 943

Liang, G. Y., Whiteford, A. D., \& Badnell, N. R. 2009b, A\&A, 500, 1263

Liang, G. Y., Badnell, N. R., Crespo López-Urrutia, J. R., et al. 2010, ApJS, 190 322

Malinovsky, L., \& Heroux, M. 1973, ApJ, 181, 1009

Manson, J. E. 1976, J. Geophys. Res., 81, 1629

Mariska, J. T. 2013, Sol. Phys., 282, 629

Neupert, W. M., \& Kastner, S. O. 1983, A\&A, 128, 181

Storey, P. J., \& Zeippen, C. J. 2010, A\&A, 511, A78

Storey, P. J., Mason, H. E., \& Young, P. R. 2000, A\&AS, 141, 285

Storey, P. J., Zeippen, C. J., \& Le Dourneuf, M. 2002, A\&A, 394, 753

Storey, P. J., Del Zanna, G., Mason, H. E., \& Zeippen, C. 2005, A\&A, 433, 717

Tayal, S. S., \& Zatsarinny, O. 2011, ApJ, 743, 206

Thomas, R. J., \& Neupert, W. M. 1994, ApJS, 91, 461

Wang, T., Thomas, R. J., Brosius, J. W., et al. 2011, ApJS, 197, 32

Woods, T. N., Chamberlin, P. C., Harder, J. W., et al. 2009, Geophys. Res. Lett., 36,1101

Young, P. R. 2009, ApJ, 691, L77

Young, P. R., Landi, E., \& Thomas, R. J. 1998, A\&A, 329, 291

Young, P. R., Del Zanna, G., Mason, H. E., et al. 2007, PASJ, 59, 857

Young, P. R., Doschek, G. A., Warren, H. P., \& Hara, H. 2013, ApJ, 766, 127

Zeng, J., Jin, F., Zhao, G., \& Yuan, J. 2003, J. Phys. B At. Mol. Phys., 36, 3457 


\section{Appendix A: List of the observations}

Table A.1. QS observations used for the EIS calibration.

\begin{tabular}{|c|c|c|c|c|}
\hline File & Raster & Slit $(")$ & Exp. (s) & FOV $\left({ }^{\prime \prime}\right)$ \\
\hline 20061223_161013 & HPW001_FULLCCD_RAST & 1 & 90 & $128 \times 128$ \\
\hline 20070114_220819 & SYNOP001_slit & 1 & 90 & $1 \times 256$ \\
\hline 20070130_111912 & HPW001_FULLCCD_RAST & 1 & 90 & $128 \times 128$ \\
\hline 20070216_112350 & SYNOP001_slit & 1 & 90 & $1 \times 256$ \\
\hline 20070316_180127 & SYNOP001_slit & 1 & 90 & $1 \times 256$ \\
\hline 20070421000705 & SYNOP001_slit & 1 & 90 & $1 \times 256$ \\
\hline 20070517000450 & SYNOP001_slit & 1 & 90 & $1 \times 256$ \\
\hline 20070602_131520 & HPW008_FULLCCD_RAST & 1 & 25 & $128 \times 128$ \\
\hline 20070620_180835 & SYNOP001_slit & 1 & 90 & $1 \times 256$ \\
\hline 20070720_110822 & SYNOP001_slit & 1 & 90 & $1 \times 256$ \\
\hline 20070817_062935 & SYNOP001_slit & 1 & 90 & $1 \times 256$ \\
\hline 20070913_175836 & SYNOP001_slit & 1 & 90 & $1 \times 256$ \\
\hline 20071024061835 & SYNOP001_slit & 1 & 90 & $1 \times 256$ \\
\hline 20071125104656 & SYNOP001_slit & 1 & 90 & $1 \times 256$ \\
\hline 20071222 & SYNOP001_slit & 1 & 90 & $1 \times 256$ \\
\hline 20080121_160213 & SYNOP002_FUL̄LCCD & 1 & 90 & $128 \times 184$ \\
\hline 20080705_112034 & FELDMAN_QSCH_ATLASv1 & 2 & 120 & $24 \times 304$ \\
\hline $20081217 \quad 110519$ & HPW001_FULLCCD RAST & 1 & 90 & $128 \times 128$ \\
\hline 20090323_174230 & SYNOP001_slit & 1 & 90 & $1 \times 256$ \\
\hline 20090413_175041 & HPW001_FULLCCD_RAST & 1 & 90 & $128 \times 128$ \\
\hline 20090511_180929 & SYNOP001_slit & 1 & 90 & $1 \times 256$ \\
\hline 20090623_182812 & SYNOP001_slit & 1 & 90 & $1 \times 256$ \\
\hline 20090720_060035 & SYNOP001_slit & 1 & 90 & $1 \times 256$ \\
\hline 20090813_180429 & SYNOP001_slit & 1 & 90 & $1 \times 256$ \\
\hline 20090919180557 & SYNOP001_slit & 1 & 90 & $1 \times 256$ \\
\hline $20091007 \quad 120219$ & HPW001_FULLCCD RAST & 1 & 90 & $128 \times 128$ \\
\hline 20091023_060550 & SYNOP001_slit & 1 & 90 & $1 \times 256$ \\
\hline 20091113_180529 & SYNOP001_slit & 1 & 90 & $1 \times 256$ \\
\hline 20091227_063535 & SYNOP001_slit & 1 & 90 & $1 \times 256$ \\
\hline 20100501_054013 & Atlas_30 & 2 & 30 & $120 \times 160$ \\
\hline 20101008_101526 & Atlas_120 & 2 & 120 & $120 \times 160$ \\
\hline 20101220_050526 & Atlas_120 & 2 & 120 & $120 \times 160$ \\
\hline 20110413_132033 & Atlas_120 & 2 & 120 & $120 \times 160$ \\
\hline 20110603 & Atlas $060 \times 512$ 60s & 1 & 60 & $60 \times 512$ \\
\hline 20110831_054534 & Atlas_60 & 2 & 60 & $120 \times 160$ \\
\hline 20111226_181940 & Atlas_120 & 2 & 120 & $120 \times 160$ \\
\hline 20120428_151319 & Atlas_060x512_60s & 1 & 60 & $60 \times 512$ \\
\hline 20120913_182534 & Atlas_120 & 2 & 120 & $120 \times 160$ \\
\hline
\end{tabular}

Notes. The columns indicate the file name (date and UT time), the EIS raster acronym, the slit used, the exposure time, and the field of view (FOV) of the observation. 
Table A.2. AR observations used for the EIS calibration.

\begin{tabular}{|c|c|c|c|c|}
\hline File & Raster & Slit $\left({ }^{\prime \prime}\right)$ & Exp. (s) & FOV $\left({ }^{\prime \prime}\right)$ \\
\hline 20061225_221058 & SYNOP001_slit & 1 & 90 & $1 \times 256$ \\
\hline $20061225 \_225013$ & HPW001_FULLCCD_RAST & 1 & 90 & $128 \times 128$ \\
\hline 20070118_120435 & SYNOP001_slit & 1 & 90 & $1 \times 256$ \\
\hline 20070210_000119 & SYNOP001_slit & 1 & 90 & $1 \times 256$ \\
\hline 20070220_054028 & SYNOP001_slit & 1 & 90 & $1 \times 256$ \\
\hline 20070322_125559 & SYNOP001_slit & 1 & 90 & $1 \times 256$ \\
\hline 20070423_184443 & SYNOP001_slit & 1 & 90 & $1 \times 256$ \\
\hline 20070511_105544 & SYNOP001_slit & 1 & 90 & $1 \times 256$ \\
\hline 20070519_180450 & SYNOP001_slit & 1 & 90 & $1 \times 256$ \\
\hline 20070607_181319 & SYNOP001_slit & 1 & 90 & $1 \times 256$ \\
\hline 20070630_175020 & SYNOP001_slit & 1 & 90 & $1 \times 256$ \\
\hline 20070630_175235 & SYNOP001_slit & 1 & 90 & $1 \times 256$ \\
\hline 20070630_175451 & SYNOP001_slit & 1 & 90 & $1 \times 256$ \\
\hline 20070630_175706 & SYNOP001_slit & 1 & 90 & $1 \times 256$ \\
\hline 20070714_000949 & SYNOP001_slit & 1 & 90 & $1 \times 256$ \\
\hline 20070819_133227 & HPW001_FULLCCD_RAST & 1 & 90 & $128 \times 128$ \\
\hline 20070929_102529 & SYNOP001_slit & 1 & 90 & $1 \times 256$ \\
\hline 20071020_021049 & SYNOP001_slit & 1 & 90 & $1 \times 256$ \\
\hline 20071114_000707 & SYNOP001_slit & 1 & 90 & $1 \times 256$ \\
\hline 20071206_175549 & SYNOP001_slit & 1 & 90 & $1 \times 256$ \\
\hline 20080107_101448 & SYNOP001_slit & 1 & 90 & $1 \times 256$ \\
\hline 20080118_103435 & SYNOP001_slit & 1 & 90 & $1 \times 256$ \\
\hline 20080204_104700 & SYNOP001_slit & 1 & 90 & $1 \times 256$ \\
\hline 20080620_230339 & HPW001_FULLCCD_RAST & 1 & 90 & $128 \times 128$ \\
\hline 20081217_110519 & HPW001_FULLCCD_RAST & 1 & 90 & $128 \times 128$ \\
\hline 20090322_060630 & SYNOP001_slit & 1 & 90 & $1 \times 256$ \\
\hline 20090521_180529 & SYNOP001_slit & 1 & 90 & $1 \times 256$ \\
\hline 20090725_055049 & SYNOP001_slit & 1 & 90 & $1 \times 256$ \\
\hline $20091025 \_214625$ & HPW008_FULLCCD_RAST & 1 & 25 & $128 \times 128$ \\
\hline 20100123_171532 & SYNOP001_slit & 1 & 30 & $1 \times 256$ \\
\hline 20100517_135741 & Atlas_60 & 2 & 60 & $120 \times 160$ \\
\hline 20100922_112633 & Atlas_60 & 2 & 60 & $120 \times 160$ \\
\hline 20110121_123757 & Atlas_60 & 2 & 60 & $120 \times 160$ \\
\hline 20110522_103354 & Atlas_60 & 2 & 60 & $120 \times 160$ \\
\hline 20110726_175935 & Atlas_60 & 2 & 60 & $120 \times 160$ \\
\hline 20111022_100543 & Atlas_60 & 2 & 60 & $120 \times 160$ \\
\hline 20111217_125856 & Atlas_60 & 2 & 60 & $120 \times 160$ \\
\hline 20120416_124033 & Atlas_60 & 2 & 60 & $120 \times 160$ \\
\hline 20120704_223657 & Atlas_60 & 2 & 60 & $120 \times 160$ \\
\hline 20120830_234044 & Atlas_60 & 2 & 60 & $120 \times 160$ \\
\hline
\end{tabular}




\section{Appendix B: Effective areas and long-term correction}

In what follows, we provide some IDL commands to obtain the effective areas and the long-term correction within SolarSoft.

gdz_sw $=[165,171,174.5,177.2,178.1,180.4,182.2, \$$

$184.5,185.2,186.9,188.3,190, \$$

$192.4,192.8,193.5,194.7,195.1, \$$

$196.6,197.4,200,201.1,202 ., \$$

$202.7,204.9,208 ., 209.9,211.3]$

gdz_eff_sw $=\$$

$[0.000174973 / 1.5,0.000255772 / 1.5,0.00158207 / 1.5, \$$ $0.00476608 / 1.55,0.00705735 / 1.5,0.0168637 / 1.45, \$$

$0.0316499 / 1.4,0.0647319 / 1.35,0.0779082 / 1.35, \$$

$0.115240 / 1.4,0.150199 / 1.45,0.194897 / 1.25, \$$

$0.255993 / 1.13,0.264945 / 1.1,0.279607 / 1.05, \$$

$0.298884 / 1.02, \quad 0.302737 * 1 ., 0.301859 / 1.05, \$$

$0.287675 / 1.15,0.174608 * 1.05,0.119586 / 1.0, \$$

$0.0838537 / 1 ., 0.0635698 / 1 ., \quad 0.0332376 / 1.0, \$$

$0.0189209 / 1 ., 0.0133581 / 1 ., 0.0105513 / 1$.

gdz_lw $=[245 ., 252 ., 255,257 ., 259, \$$

263. , 265., 268., 270., \$

272., 274., 277., 281., 286., 292]

gdz_eff_lw $=\$$

$[0.022673 * 0.8,0.03908 * 0.75,0.05065 * 0.78, \$$

$0.0588 * 0.8,0.06738 * 0.85,0.0861 * 0.9, \$$

$0.09551 * 0.95,0.106984 * 1.0,0.110764 * 1.02, \$$

$0.10944 * 1.03, \quad 0.1026 * 1.03,0.084775 * 0.9, \$$

$0.05718 * 0.87,0.0333 * 0.85,0.01679 * 0.85] / 1.1$

; the effective area at a SW wavelength(Angstroms) ; 1 is obtained as

; eff=interpol (gdz_eff_sw , gdz_sw, w1),/spline)

; the effective area at a LW wavelength(Angstroms)

; w2 is obtained as

; eff= interpol(gdz_eff_lw , gdz_lw, w2),/spline)

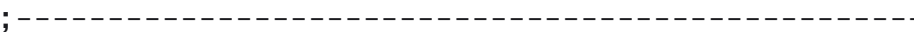

Xtime_ref=ANYTIM2TAI ('22-Sept-2006 21:36:00.000') coeff $1=[1.0326230,-5.2495791 \mathrm{e}-09,1.2055185 \mathrm{e}-17]$

; date is any observation time.

Xtime=ANYTIM2TAI (date)

; degr gives the degradation

degr=poly(Xtime - Xtime_ref, coeff1)

We note that this correction cannot be extrapolated to dates later than September 2012.

\section{Appendix C: The Si VII vs. Fe VIII problem}

We have calculated the contribution functions $G(T)$ of a selection of TR lines observed by Hinode/EIS, using CHIANTI v.7.1 data (Landi et al. 2012), in particular the new ion abundances (in ionisation equilibrium). They are shown in Fig. C.1 (top). Any small differences in the contribution functions of two lines in the transition region, where there is a steep variation in temperature, can have a large effect (see, for example, the large effects on active region loops in Del Zanna 2003).

To estimate the effects that a steep variation in temperature can have, we have taken a QS DEM obtained from the on-disk EIS observations of 2006 Dec 23, and folded it with the $G(T)$ of a few main EIS lines, as an example. They are shown in Fig. C.1 (bottom). It is clear that the emission in the Fe VIII and Si VII has two main components, one coronal (1 MK) and one in the lower transition region. It is also clear that the TR component of Fe VIII should have a similar response to that of Mg VI, and not Si VII. It is fair to say, however, that for both Fe VIII and Si VII a significant contribution is predicted to originate from the coronal component. This is of course assuming that a continuous distribution of plasma between TR temperatures and the corona exists. The TR Si VII component accounts for almost $30 \%$ of the intensity of the line in the $\log T[\mathrm{~K}]=5.2-5.9$ range, while the Fe VIII TR component accounts for over 40\%; Mg VI is clearly a pure TR line.
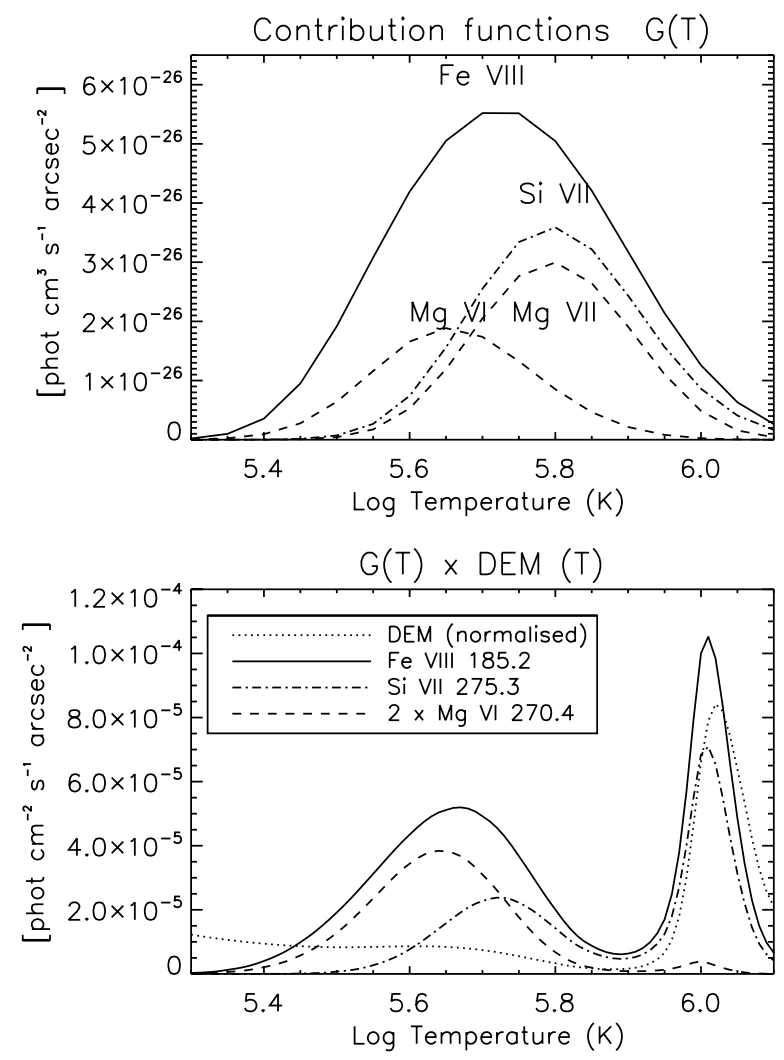

Fig. C.1. Top: contribution functions $G(T)$ of a selection of TR lines observed by Hinode/EIS, using CHIANTI v.7.1 data. Bottom: the same $G(T)$ values multiplied for a QS DEM. We note that the $G(T) \times$ $\operatorname{DEM}(T)$ of $\mathrm{Mg}$ VI was increased by a factor of two. 